\title{
Dry and Semidry Tropical Cyclones $\mathscr{O}$
}

\author{
TIMOTHY W. CRONIN \\ Department of Earth, Atmospheric, and Planetary Sciences, Massachusetts Institute of Technology, Cambridge, Massachusetts \\ DANIEL R. CHAVAS \\ Department of Earth, Atmospheric, and Planetary Sciences, Purdue University, West Lafayette, Indiana
}

(Manuscript received 11 December 2018, in final form 25 April 2019)

\begin{abstract}
It is widely believed that tropical cyclones are an intrinsically moist phenomenon, requiring evaporation and latent heat release in cumulus convection. Recent numerical modeling by Mrowiec et al., however, challenged this conventional wisdom by finding the formation of axisymmetric dry tropical cyclones in dry radiative-convective equilibrium (RCE). This paper addresses ensuing questions about the stability of dry tropical cyclones in 3D, the moist-dry vortex transition, and whether existing theories for intensity, size, and structure apply to dry cyclones. A convection-permitting model is used to simulate rotating 3D RCE, with surface wetness (0-1) and surface temperature (240-300 K) smoothly varying between dry and moist states. Tropical cyclones spontaneously form and persist for tens of days in both moist and dry/cold states, as well as part of the relatively moist/warm intermediate parameter space. As the surface is dried or cooled, cyclones weaken, both in absolute terms and relative to their potential intensities. Dry and semidry cyclones have smaller outer radii but similar-sized or larger convective centers compared to moist cyclones, consistent with existing structural theory. Strikingly, spontaneous cyclogenesis fails to occur at moderately low surface wetness values and intermediate surface temperatures of 250-270 K. Simulations with time-varying surface moisture and sea surface temperatures indicate this range of parameter space is a barrier to spontaneous genesis but not cyclone existence. Dry and semidry tropical cyclones in rotating RCE provide a compelling model system to further our understanding of real moist tropical cyclones.
\end{abstract}

\section{Introduction}

In Earth's present climate, tropical cyclones are intrinsically tied to water. Forming and intensifying almost exclusively over warm tropical oceans, tropical cyclones are most vividly depicted by satellite images of spiraling clouds—often punctuated by a cloudless eye-or by surface images of looming rainbands or rain-thrashed palm trees, and their most severe damage is caused not directly by their intense swirling winds, but by the ways that cyclones move water via storm surge and torrential rain. It is unsurprising, then, that historical study of tropical cyclones has emphasized the central role of water, particularly the energetic role played by latent heating due to water vapor condensing in deep moist convection.

Supplemental information related to this paper is available at the Journals Online website: https://doi.org/10.1175/JAS-D-180357.s1.

Corresponding author: Timothy W. Cronin, twcronin@mit.edu
The overarching goal of this paper is to challenge this notion that moisture is essential for tropical cyclones under a broader set of ambient atmospheric conditions than are presently found on Earth. Extending previous axisymmetric work by Mrowiec et al. (2011), we explore the moist-dry transition of convective vortices in threedimensional (3D) simulations of rotating radiativeconvective equilibrium.

Throughout the paper, we will use the term "tropical cyclone" to refer to a quasi-symmetric, balanced warmcore vortex, with horizontal extent much larger than vertical extent, surface cyclonic flow speeds exceeding the horizontal flow speeds associated with random convection, and vorticity deriving from planetary rotation. This definition is agnostic regarding the surface over which the cyclone exists (it may be water or a dry surface, at any temperature), and does not prescribe a specific wind speed threshold.

Early theoretical and modeling studies all reasonably invoked the role of moist convection in permitting air in the eyewall of a cyclone to ascend through an ambient 
atmosphere with stable dry stratification, leading to a warm core with concentrated vorticity and balanced cyclonic flow at lower levels (Malkus and Riehl 1960; Charney and Eliassen 1964; Ooyama 1969). The need to parameterize the upward mass and energy fluxes associated with moist convection based on azimuthal-mean cyclone-scale variables, however, led these studies to different conclusions regarding the role of moist convection in cyclone intensification and intensity-with concomitant differences in their implications for the possibility of dry convective vortices akin to tropical cyclones. Charney and Eliassen (1964) hypothesized that ambient conditional instability allowed moist convective ascent to intensify a cyclone without the need for an energy source from surface enthalpy fluxes. This extraction of available energy from a moist-unstable ambient atmosphere at large scales was termed "conditional instability of the second kind" (CISK) and would have no analog in a purely dry atmosphere. Malkus and Riehl (1960), on the other hand, noted that the magnitude of the central pressure drop associated with an intense tropical cyclone could not be generated purely by convection: ambient boundary layer equivalent potential temperatures are too low to account for the warmth observed aloft in the cores of cyclones. They reasoned that a surface enthalpy source must increase the equivalent potential temperature of the inflow, feasibly allowing for a steady-state tropical cyclone over a warm-enough ocean without any environmental conditional instability. This conclusion was supported by subsequent modeling work of Ooyama (1969), and forms the basis for much of our current theory on tropical cyclones as slantwise-neutral axisymmetric vortices, including predictions about limits on cyclone intensity (Emanuel 1986; Bister and Emanuel 1998; Tang and Emanuel 2010) and the radius-height structure of temperature and winds (Emanuel and Rotunno 2011; Chavas et al. 2015, hereafter C15). Although this axisymmetric and thermodynamic paradigm has proven useful, it does not explain features such as azimuthal asymmetries (Shea and Gray 1973; Schubert et al. 1999; Montgomery et al. 2006) and boundary layer shocks (Williams et al. 2013; Kepert 2013; Abarca and Montgomery 2014).

The concept of tropical cyclones as balanced slantwise-neutral convective vortices-with inflowing air simultaneously diminished in angular momentum by surface friction and augmented in moist entropy by surface enthalpy fluxes-is entirely compatible with the possibility of dry or semidry tropical cyclones over a surface that is primarily warm, rather than moist, relative to the near-surface atmosphere. Study of dry convective vortices has drawn from the theory of moist hurricanes in the context of dust devils (Rennó et al. 1998) and Martian dust storms (Rafkin 2009), but both of these situations differ qualitatively from tropical cyclones. The rotation of dust devils derives from randomsigned local vorticity anomalies associated with wind shear rather than planetary rotation, and the radiativedynamic dust feedback mechanism proposed by Rafkin (2009) is associated with flow-dependent interior, rather than boundary, entropy sources. Mrowiec et al. (2011) explicitly explored the possibility of dry tropical cyclones, using an axisymmetric atmospheric model in both dry and moist configurations, and found that dry cyclones readily formed in an atmosphere with dry adiabatic stratification but with a surface much warmer than the near-surface atmosphere. Furthermore, the structure of these cyclones was well described by slantwise convective neutrality, and their maximum azimuthal wind speeds were found consistent with the potential intensity theory of Emanuel (1986).

The findings of Mrowiec et al. (2011) are striking, yet have spurred little follow-up work. In our view, the most notable research gaps are the lack of 3D simulations of dry tropical cyclones, and the lack of exploration of the intermediate parameter space between dry and moist limits. The former is a particular concern because axisymmetric models are inherently biased toward forming vortices, and lack axially asymmetric instabilities that might disrupt real 3D vortices. In this paper, we use 3D simulations of rotating radiative-convective equilibrium (RCE) with a convection-permitting atmospheric model to address the following questions:

- Do dry tropical cyclones, as found in axisymmetry by Mrowiec et al. (2011), form and persist in 3D RCE?

- Is the dry limit singular, or is the moist-to-dry transition continuous?

- Does existing potential intensity theory (for maximum azimuthal wind speeds) also provide an upper bound on winds for dry and semidry tropical cyclones?

- How does storm size differ for moist, dry, and semidry storms?

- Does existing theory for storm structure apply to dry and semidry tropical cyclones?

The reader likely has already inferred that our answer to the first question is an enthusiastic "yes!," as this paper would have a quite different title and introduction had we found no stable 3D dry tropical cyclones in our simulations. The answers to the other questions are more nuanced, and we will explore them at length below.

We choose 3D rotating RCE as our model configuration because it represents the simplest equilibrated climate state in which tropical cyclones can form and interact with one another. Most studies of rotating RCE with either parameterized or resolved convection have 
found spontaneous tropical cyclogenesis: that is, one or more cyclones (with number dependent on domain size, Coriolis parameter, sea surface temperature, and other properties of the simulation; e.g., Zhou et al. 2014) spontaneously emerge from spatially random convection given a large-enough domain and long-enough simulations (Khairoutdinov and Emanuel 2013; Wing et al. 2016; Muller and Romps 2018). Tropical cyclones in rotating RCE are generally long-lived and stable, select their own distribution of sizes and intensities, and tend to fill the domain if enough space is available (Chavas and Emanuel 2014; Khairoutdinov and Emanuel 2013; Zhou et al. 2014).

The primary motivation of this work is to improve our understanding of real moist tropical cyclones by removing the complicating influences of moisture, thus filling a gap in our model hierarchy of tropical cyclones (e.g., Held 2005). Dry tropical cyclones may also connect with real planetary climate in several ways. First, in colder climates of Earth's past, moisture would likely play a smaller role in any tropical cyclones that did form, potentially making such storms "drier" in character. Second, weak constraints on the atmospheric composition, volatile species, and surface properties on early Earth serve as a motivation for expanding the bounds of parameter space that have been explored by previous simulation of tropical cyclones. Third, hurricane-like features may exist on other worlds (e.g., Tokano 2013), including the rapidly growing tableau of planetary atmospheres outside the solar system. From a standpoint of fundamental science, dry tropical cyclones offer the possibility of linking study of hurricanes to the vast body of experimental, modeling, and theoretical work on dry convection that has been built over the past century.

We begin by describing the simulation design (section 2a), our storm identification and tracking methods (section 2b), definitions of generalized potential intensity (section $2 \mathrm{c}$ ), and storm size (section $2 \mathrm{~d}$ ). We then present and discuss our results, starting with nonrotating dry-to-moist radiative-convective equilibrium (section $3 \mathrm{a})$, continuing with the contrasting properties of moist, half-dry, and dry cyclones (sections $3 \mathrm{~b}$ and $3 \mathrm{c}$ ), then examining the moist-dry transition in more detail (sections $3 \mathrm{~d}$ and $3 \mathrm{e}$ ), and finishing with an analysis of the radial structure of azimuthal winds (section 3f). We conclude the paper in section 4 by discussing the limitations of this study and possible directions for future work.

\section{Methods}

\section{a. Simulation design}

We use the System for Atmospheric Modeling (SAM, version 6.10.8, Khairoutdinov and Randall 2003) convectionpermitting model to simulate the atmosphere in a doubly periodic domain over a surface of constant temperature $T_{S}$. We modify the code to use a simple radiative cooling scheme similar to Chavas and Emanuel (2014), with constant cooling if the temperature $T$ exceeds a threshold $T_{T}$, and Newtonian relaxation toward the threshold otherwise:

$$
(\partial T / \partial t)_{\mathrm{rad}}=\left\{\begin{array}{cc}
-\dot{Q}, & T>T_{T}, \\
\left(T_{T}-T\right) / \tau, & T \leq T_{T}
\end{array}\right.
$$

In all simulations, we take $\dot{Q}=1 \mathrm{~K} \mathrm{day}^{-1}$, and $\tau=$ 20 days.

We also modify the code to simplify the bulk formulae for surface sensible $(H)$ and latent $(E)$ heat fluxes by removing the dependence of heat and momentum exchange coefficients on wind speed or near-surface stability:

$$
\begin{aligned}
& H=\rho c_{K} c_{p} V_{S}\left(T_{S}-\theta_{1}\right), \\
& E=\rho c_{K} L_{v} V_{S}\left(\beta q_{S}^{*}-q_{1}\right),
\end{aligned}
$$

where $\rho$ is the density of the near-surface air, $c_{K}=1.5 \times 10^{-3}$ is a constant enthalpy exchange coefficient, $c_{p}$ is the specific heat of air at constant pressure, $\theta_{1}$ is the potential temperature of the lowest model level, $L_{v}$ is the latent heat of vaporization of water, $q_{S}^{*}$ is the saturation specific humidity at the surface, and $q_{1}$ is the specific humidity of the lowest model level. We refer to the parameter $\beta(0 \leq \beta \leq 1)$ in Eq. (3) as the surface wetness, as it reduces the specific humidity of the surface below that of pure water: $\beta=1$ represents a completely moist surface and $\beta=0$ represents a completely dry surface. The parameter $\beta$ is identical to the activity coefficient of water in the literature about hydrology of saline water bodies; the average value of $\beta$ for ocean water is about 0.97 (Salhotra et al. 1985), but values as low as 0.4 have been measured for the Dead Sea (Oroud 1994). Physically, the saturation specific humidity over a liquid surface is reduced by a simple multiplicative factor if water is only one of several components of the liquid. For an ideal solution composed of water and another nonvolatile liquid, $\beta$ would equal the mole fraction of water in the solution (glycerol may be a liquid with such properties: it has volatility of only $\sim 1$-ppm saturation mixing ratio at $30^{\circ} \mathrm{C}$ and is highly miscible with water).

For simplicity, the drag coefficient $c_{D}=1.5 \times 10^{-3}$ is taken to be equal to the enthalpy exchange coefficient $c_{K}$. The bulk formulas [Eqs. (2) and (3)] use an effective surface wind speed $V_{S}$ that is equal to the resolved lowest model level wind speed $\left(u_{1}^{2}+v_{1}^{2}\right)^{1 / 2}$, added in quadrature with a minimum wind speed $v_{*}$, which is taken as $1 \mathrm{~m} \mathrm{~s}^{-1}$ : 


$$
V_{S}=\left(u_{1}^{2}+v_{1}^{2}+v_{*}^{2}\right)^{1 / 2} .
$$

In this paper, we focus on two sets of simulations that shift the surface turbulent heat transfer from dominantly latent to sensible heat fluxes, smoothly varying from moist to dry radiative-convective equilibrium.

1) Our first set of 16 simulations directly reduces the surface wetness at constant $T_{S}=300 \mathrm{~K}$ and $T_{T}=210 \mathrm{~K}$ by using values of $\beta$ of $0,0.001,0.003,0.01,0.03,0.1$, $0.15,0.2,0.3,0.4,0.5,0.6,0.7,0.8,0.9$, and 1.0. Because $\beta$ is a multiplier on the surface specific humidity rather than the difference between the surface and atmospheric specific humidities, low values of $\beta$ can result in a downward transfer of latent heat from the moist atmosphere into the surface.

2) Our second set of 7 simulations holds $\beta=1$, but uses surface temperatures $T_{S}$ of 300, 290,280, 270, 260, 250 , and $240 \mathrm{~K}$. For values of $T_{S} \lesssim 260 \mathrm{~K}$, the surface sensible heat flux dominates the latent heat flux, and the stratification of the troposphere is nearly dry adiabatic. In these simulations, we use $T_{T}=0.7 T_{S}$, which maintains a constant thermodynamic efficiency in the absence of dissipative heating, and also ensures that the tropopause remains at nearly the same pressure across a range of surface temperatures. Because the radiative cooling scheme described in Eq. (1) gives an integrated tropospheric radiative cooling proportional to the mass of the troposphere, the condition of nearly fixed tropopause pressure with colder surface temperatures also ensures that in radiative-convective equilibrium, the total surface enthalpy flux varies little with surface temperature. This is an unfamiliar limit, because we normally assume tropospheric radiative cooling increases with surface warming (e.g., Allen and Ingram 2002).

All simulations in these two main sets used a domain $1152 \mathrm{~km} \times 1152 \mathrm{~km}$ in the horizontal, with $2-\mathrm{km}$ horizontal grid spacing, and a Coriolis parameter $f=3 \times 10^{-4} \mathrm{~s}^{-1}$. A stretched grid was used in the vertical, with 64 levels in the lowest kilometer and a spacing of $500 \mathrm{~m}$ above $3.5 \mathrm{~km}$, and the model top at $28 \mathrm{~km}$. Simulations were run for 70 days, and each was initialized using a sounding from the last 20 days of a 100-day simulation of nonrotating RCE in a small domain $192 \mathrm{~km} \times 192 \mathrm{~km}$ (with the same resolution), to which a small amount of random thermal noise was added in the lowest model levels to initiate convection. These initialization simulations were each performed with the matching value of $\beta$ (for the first set of simulations) or of $T_{S}$ and $T_{T}$ (for the second set). Below (section 3a) we present thermodynamic profiles from these initialization simulations.
We conducted two further simulations after finding that the $\beta=1$ and $\beta=0.9$ simulations culminated with only a single vortex in the domain. This state of only a single simulated vortex in the domain was termed "lattice" equilibrium by Zhou et al. (2014) in contrast to the "turbulent" equilibrium obtained with multiple storms in the domain. They found that that lattice equilibria resulted in storms that were strongly influenced by the domain size, being either too weak or intermittent for small domains, or too large for large domains, relative to turbulent equilibrium. Sharing this view that the turbulent multistorm TC-world provides a more faithful simulation of the statistical properties of vortices (should they form), we reran the $\beta=1$ and $\beta=0.9$ simulations with larger domain sizes of $1728 \mathrm{~km} \times 1728 \mathrm{~km}$, which led to turbulent multistorm TC-worlds for all times following genesis. Thus, in our results and discussion below we focus exclusively on these larger-domain simulations; we designate them with an asterisk as the $\beta=1.0^{*}$ (equivalently $T_{S}=300 \mathrm{~K}^{*}$ ) and $\beta=0.9^{*}$ simulations, to remind the reader that they were conducted with a domain size different from the rest. We touch on a few differences between simulations with large and normal domain sizes in section $3 \mathrm{~d}$.

Snapshots at day 43 of a subset of the simulations with varied $\beta$ show that the surface wind field is dominated in most simulations (including moist and dry limits) by multiple cyclones, but that one nearly dry simulation with $\beta=0.1$ has little large-scale flow (Fig. 1). Figure 1 immediately gives several preliminary answers to the motivating questions in the introduction: the moist-dry transition appears to have both continuous and discontinuous aspects, and storm size and intensity appear to decrease with surface drying. Less glib answers, however, require us to automate vortex identification; we proceed to describe that process.

\section{b. Storm tracking and analysis}

Tropical cyclones are identified at each time step as follows. First, the surface pressure perturbation $p^{\prime}$ is calculated from the surface pressure field by subtracting the domain-mean value: $p^{\prime}(x, y)=p(x, y)-\bar{p}$. Grid points with $p^{\prime} \geq-5 \mathrm{hPa}$ are masked out to isolate regions of relatively low pressure. This field is then replicated in a $3 \times 3 x-y$ grid to allow for identification of regions that cross horizontal boundaries of the doubly periodic domain. Contiguous low pressure objects are created using an eight-connected component labeling algorithm, and objects containing at least 13 grid points are retained. For each object, the storm center is defined as the pressure-weighted centroid of the 13 grid points with the lowest $p^{\prime}$ values, such that the center will be located in the vicinity of the local minimum pressure 

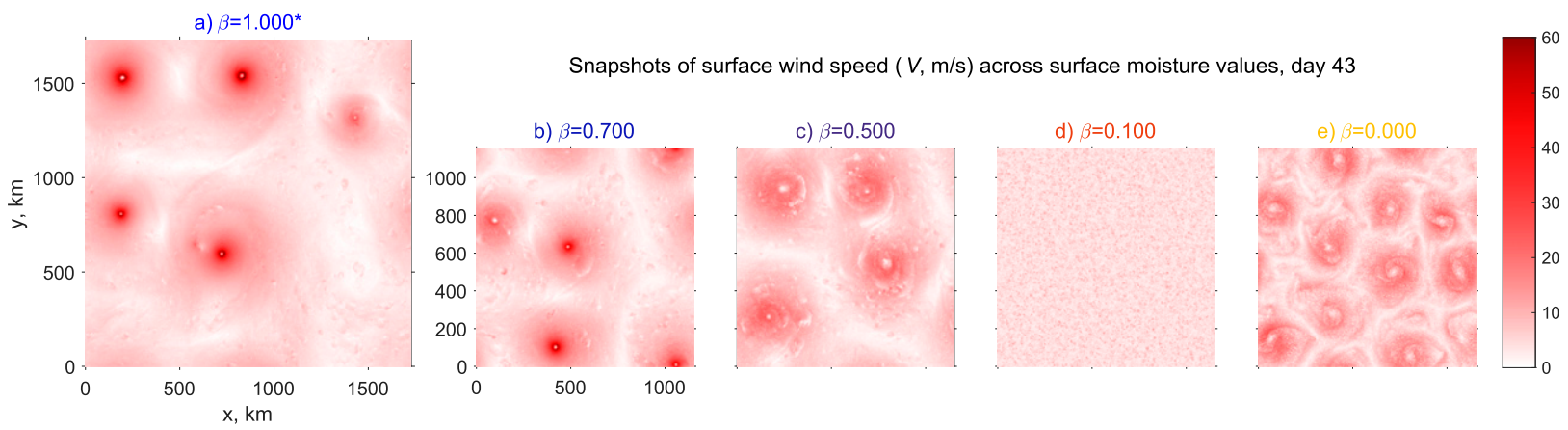

FIG. 1. Snapshots at day 43 of the surface wind speed $V\left(\mathrm{~m} \mathrm{~s}^{-1}\right)$ for several simulations with varied surface wetness: (a) $\beta=1^{*},(\mathrm{~b}) \beta=0.7$, (c) $\beta=0.5$, (d) $\beta=0.1$, and (e) $\beta=0$.

while minimizing noise due to small scale variability at one or two grid points. The storm central pressure is defined by bilinearly interpolating the pressure field to the storm center. Finally, for each storm center, radiusheight cross sections of relevant azimuthally averaged quantities are extracted from the data, including the decomposition of the total wind field into its radial and tangential components. Azimuthal averages are calculated in $\Delta r=2 \mathrm{~km}$ radial bins moving outwards beginning at $r=1 \mathrm{~km}$ in 2-km steps. Examples of this process for $\beta=1^{*}, \beta=0.5$, and $\beta=0$ are shown later (see Fig. 4) and will be discussed in detail in section $3 b$.

When discussing winds in the context of cyclones, we will use a cylindrical-polar decomposition, with $u$ as the outward radial wind component, $v$ the counterclockwise (here, cyclonic) tangential wind component, and $w$ the vertical wind component. We will use $v_{m}$ to denote the maximum azimuthally averaged tangential wind speed, $r_{m}$ as the radius of maximum winds, and capital $V$ to indicate horizontal wind speed $\left[\left(u^{2}+v^{2}\right)^{1 / 2}\right]$. Unless otherwise stated, values refer to winds at the lowest model level $(z=37.5 \mathrm{~m})$.

\section{c. Potential intensity}

We define a simplified potential intensity $v_{p}$-generalizable across the range of surface wetness-based on the domain-average sounding:

$$
v_{p}^{2}=\underbrace{\frac{c_{K}}{c_{D}}}_{\text {Exchange coefficient ratio }} \times \overbrace{\frac{T_{S}-T_{T}}{T_{S}}}^{\text {Thermodynamic efficiency }} \times \underbrace{\left[\left(c_{p} T_{S}+\beta L_{v} q_{S}^{*}\right)-\left(c_{p} T_{1}+L_{v} q_{1}\right)\right]}_{\text {Enthalpy disequilibrium }} .
$$

This expression is a product of three factors: a ratio of exchange coefficients (always 1 in our simulations), a thermodynamic efficiency, and an enthalpy disequilibrium between the surface and lowest atmospheric level, modified by surface wetness. Because SAM does not include heating due to kinetic energy dissipation, the denominator of the Carnot efficiency remains $T_{S}$ rather than $T_{T}$ (Bister and Emanuel 1998). Since storm structure and size may vary between the moist and dry limits, and along with it the relationship between central pressure deficit and cyclone intensity (Chavas et al. 2017), we evaluate $q_{S}^{*}$ at the domain-average surface pressure, rather than iterating to calculate $q_{S}^{*}$ consistent with the minimum attainable central pressure of a cyclone. Thus, we exclude the feedback between reduced central pressure and increased $q_{S}^{*}$ which increases the surface enthalpy disequilibrium for moist storms. Since our potential intensities are typically only $\sim 60 \mathrm{~m} \mathrm{~s}^{-1}$, this missing feedback does not greatly underestimate the "true" potential intensity; its value increases by only $10 \mathrm{~m} \mathrm{~s}^{-1}$ with the pressure dependence effect included using the Emanuel code (v2017-07-25; ftp://texmex.mit.edu/ pub/emanuel/TCMAX/) for a comparable moist adiabatic sounding over a moist surface without the effect of dissipative heating.

\section{d. Storm size}

We use three metrics of storm size. The first two, $r_{0}$ and $r_{8}$, indicate the radius for a given storm where the lowest-level azimuthal mean tangential wind $v(r)$ first passes below 0 or $8 \mathrm{~m} \mathrm{~s}^{-1}$, respectively. To find these, we opt for the simplest method of selecting the first radius greater than $r_{m}$ where $v(r)<0$ or $v(r)<8 \mathrm{~m} \mathrm{~s}^{-1}$ for each 

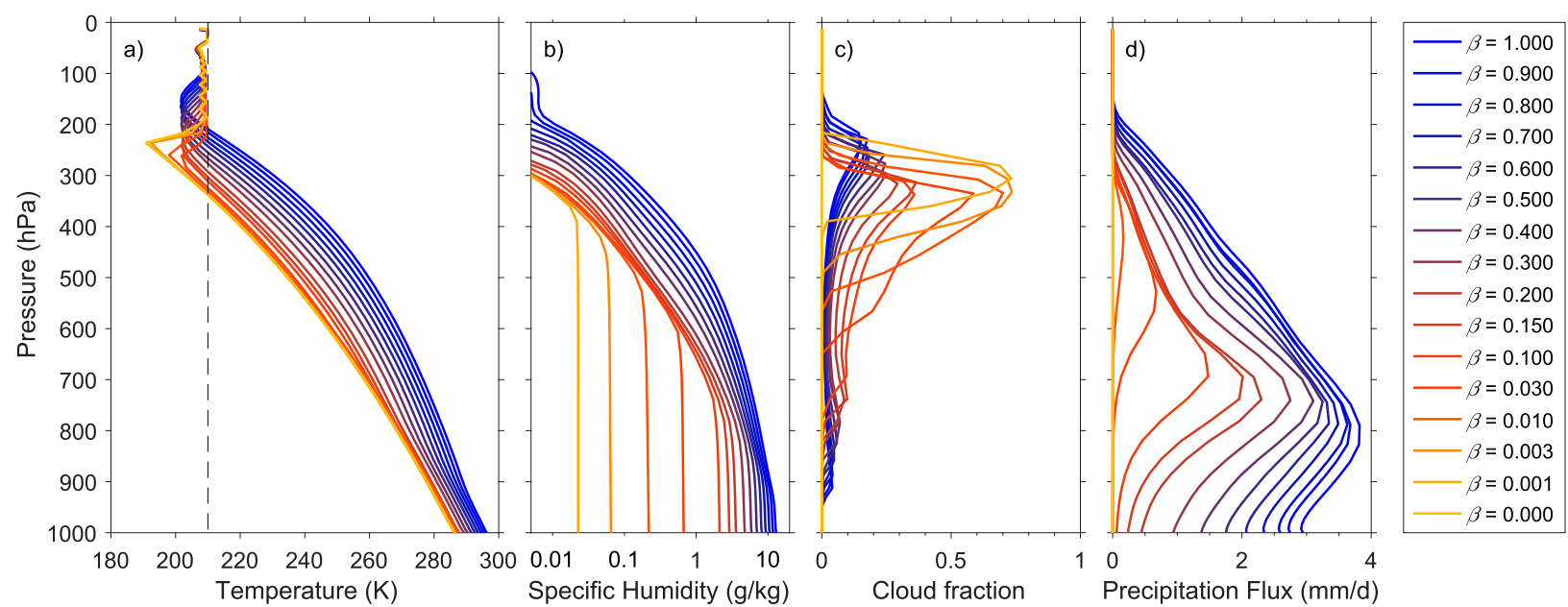

FIG. 2. Profiles from nonrotating initialization simulations with varied surface wetness $\beta$ for (a) temperature, (b) specific humidity (note logarithmic horizontal axis), (c) cloud fraction, and (d) precipitation flux. The surface temperature $T_{S}=300 \mathrm{~K}$ in all simulations, and the radiative tropopause temperature $T_{T}=210 \mathrm{~K}$, indicated with a vertical dashed line in (a). The depth of the well-mixed dry adiabatic subcloud layer can be inferred from the portion of the troposphere in each simulation where the specific humidity is constant with height in (b); lowest cloud bases in (c) coincide with the top of the subcloud layer. Note that specific humidity, cloud fraction, and precipitation flux are 0 at all heights in the $\beta=0$ simulation.

storm. If a storm has no radius where the circulation first reverses (becoming anticyclonic), we set $r_{0}$ to the maximum attainable radius in the domain $(L \sqrt{2} / 2$; this occurs for fewer than $0.04 \%$ of identified vortices). This simple method has limitations, because radial decay of winds outside $r_{m}$ is nonmonotonic for many storms, especially those involved in strong cyclone-cyclone interaction pairs or triads. More sophisticated methods that involve fitting the outer wind profile with a theoretical curve, however, are still subject to errors from vortex-vortex interactions, and require additional assumptions.

The third metric, $r_{C}$, is based on the theoretical radius where tangential winds first cross zero for a fully convective, slantwise-neutral vortex constrained by selfstratifying outflow, as described in Emanuel and Rotunno (2011). Although they refer to this value as $r_{0}$, the outer winds in simulated storms decay more slowly than their model predicts, due to the lack of convective coupling of the boundary layer and free troposphere at large radii. Because of this mismatch, we distinguish between the theoretical fully convective vortex size of Emanuel and Rotunno (2011), $r_{C}$, and the radius at which winds actually vanish, $r_{0}$. Using our terminology, it is $r_{C}$ and not $r_{0}$ that should be bounded above by $v_{p} / f$ (as noted in Emanuel 2004), because the derivation of this upper bound is valid only in convectively coupled regions of a cyclone. An approximate value for $r_{C}$ is given by simplifying Eqs. (36) and (37) of Emanuel and Rotunno (2011) in the specific case $c_{K} / c_{D}=1$, using the definition of an inner-core Rossby number Ro $\equiv v_{m} / f r_{m}$, and without making the cyclostrophic simplification that the angular momentum at the radius of maximum winds is dominated by $v_{m} r_{m}$ relative to $f r_{m}^{2} / 2$ :

$$
r_{C} \approx r_{m} \sqrt{4 \mathrm{Ro}+1} .
$$

Note that the derivation of Eq. (37) of Emanuel and Rotunno (2011) is valid only in the limit that $R o \gg 1$, so the true extent of a fully convective vortex may differ from this expression if Ro $\sim 1$ (no analytic solution is known for Ro $\sim 1$ ).

\section{Results and discussion}

\section{a. Nonrotating RCE from moist to dry limits}

Temperature and humidity profiles from the varied$\beta$ initialization simulations show that as the surface becomes drier, the dry adiabatic subcloud layer at the bottom of the troposphere grows upward. The moistadiabatic cloud layer, which occupies most of the troposphere for $T_{S}=300 \mathrm{~K}$ and $\beta=1$, shrinks with surface drying, vanishing entirely for $\beta=0$ (Fig. 2).

Surprisingly, the cloud fraction increases as the surface is dried (Fig. 2c); for surfaces with $\beta>0.2$ the effects of surface moistening are reminiscent of the decrease and upward shift in high cloud amount with atmospheric warming noted by Bony et al. (2016) in climate models and in SAM by Cronin and Wing (2017). For very dry surfaces, the entire troposphere resembles a boundary layer topped with broken nonprecipitating clouds. For $\beta=0.001$ and $\beta=0.003$, the autoconversion 

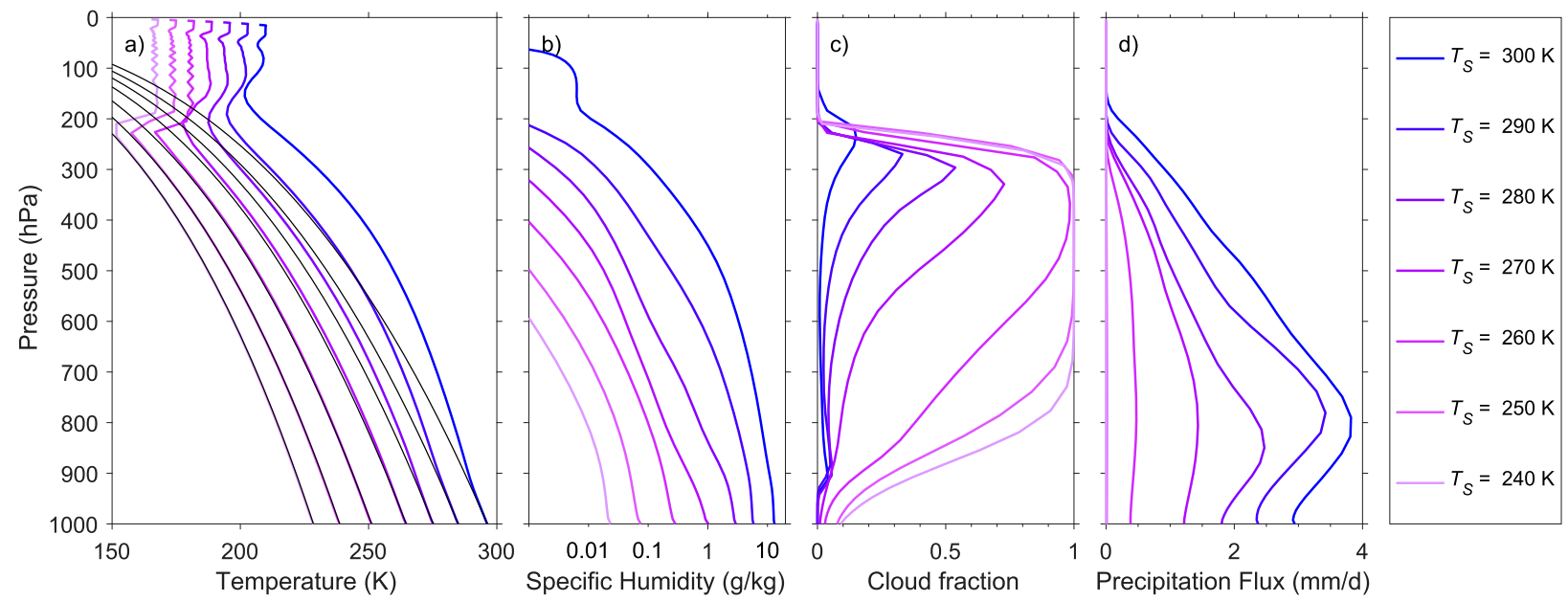

FIG. 3. As in Fig. 2, but for nonrotating initialization simulations with varied surface temperature. Black solid lines in (a) indicate dry adiabats starting at the surface pressure and average surface air temperature of each of the seven simulations.

threshold for ice clouds $\left(0.1 \mathrm{~g} \mathrm{~kg}^{-1}\right)$ exceeds specific humidities in the subcloud layer, so precipitation cannot form even if surface parcels are lifted high enough that saturation specific humidity is zero. In these simulations, we speculate that the entire layer above the lifted condensation level would fill with cloud were it not for ice cloud sedimentation. Two other notable features of dry and semidry RCE are the sharp tropopause inversion in the dry limit (Fig. 2a), and the vigorous internal moisture recycling for moderately dry surfaces implied by precipitation fluxes which have lower-tropospheric values several times as large as those at the surface (Fig. 2d). Upper-tropospheric temperatures well below the radiative tropopause temperature $\left(T_{T}=210 \mathrm{~K}\right)$ indicate the presence of an "overshooting layer" that is convectively cooled and radiatively heated (similar to Pauluis and Held 2002).

The warm-cold transition is not marked by a similar increase in the depth of the subcloud layer or decrease in precipitation efficiency (Fig. 3). Instead the cold limit is marked by convection that is energetically dry-with dry adiabatic stratification and dominantly sensible heat transport-but which carries water vapor and cloud ice with it as a nearly passive tracer. For surface temperatures of 240-260 K, the bulk thermal stratification of the troposphere is less than $1 \mathrm{~K}$, the troposphere is saturated and overcast over a deep layer, and the tropopause inversion is sharp-as in the small- $\beta$ limit (Figs. 3a,c). In the cold limit, the subcloud layer seems to vanish, as clouds exist all the way down to the surface, and the distinction between dry and moist adiabatic stratification disappears. The behavior of cloud and relative humidity fields in the cold limit is undoubtedly sensitive to the treatment of ice microphysics, but with energetically dry convection and prescribed radiative cooling, it is unclear whether this alters convective dynamics.

The transition from moist to dry nonrotating RCE appears to be rich territory for further study, particularly for understanding controls on tropopause temperature structure, cloud fraction, and how precipitation evaporation affects rainfall rates over land. Deeper analysis of the nonrotating moist-dry transition, however, is deferred to future work: rotating RCE will be the focus of the remainder of this paper.

\section{b. Storm basics: Moist, half-dry, dry}

As a primary concern of our study is the transition between moist and dry rotating RCE, we begin with a detailed comparison of simulations with $\beta=1^{*}, \beta=0.5$, and $\beta=0$ (all with $T_{S}=300 \mathrm{~K}$ and $T_{T}=210 \mathrm{~K}$; see Figs. 1a,c,e). For brevity, we refer to these as "moist," "half-dry," and "dry" simulations, respectively.

In all three simulations, cyclones spontaneously emerge from initially random convection; we compare cyclone structure from an illustrative snapshot 43 days into each simulation (see supplemental videos S1-S3 for time evolution of surface wind speeds). As previously noted (Figs. 1a,c,e), Figs. 4a-c show that cyclones appear to fill the domain in the three simulations, as in previous work on moist rotating RCE (e.g., Zhou et al. 2014). The dry simulation achieves this result with a larger density of smaller cyclones $\left(\sim 7.5\right.$ per 1 million $\left.\mathrm{km}^{2}\right)$ than either the half-dry $\left(\sim 3\right.$ per 1 million $\left.\mathrm{km}^{2}\right)$ or moist $(\sim 1.3$ per 1 million $\mathrm{km}^{2}$ ) simulations.

Radius-height cross sections of the tangential wind and temperature perturbation for example storms from moist (Fig. 4d), half-dry (Fig. 4e) and dry (Fig. 4f) 

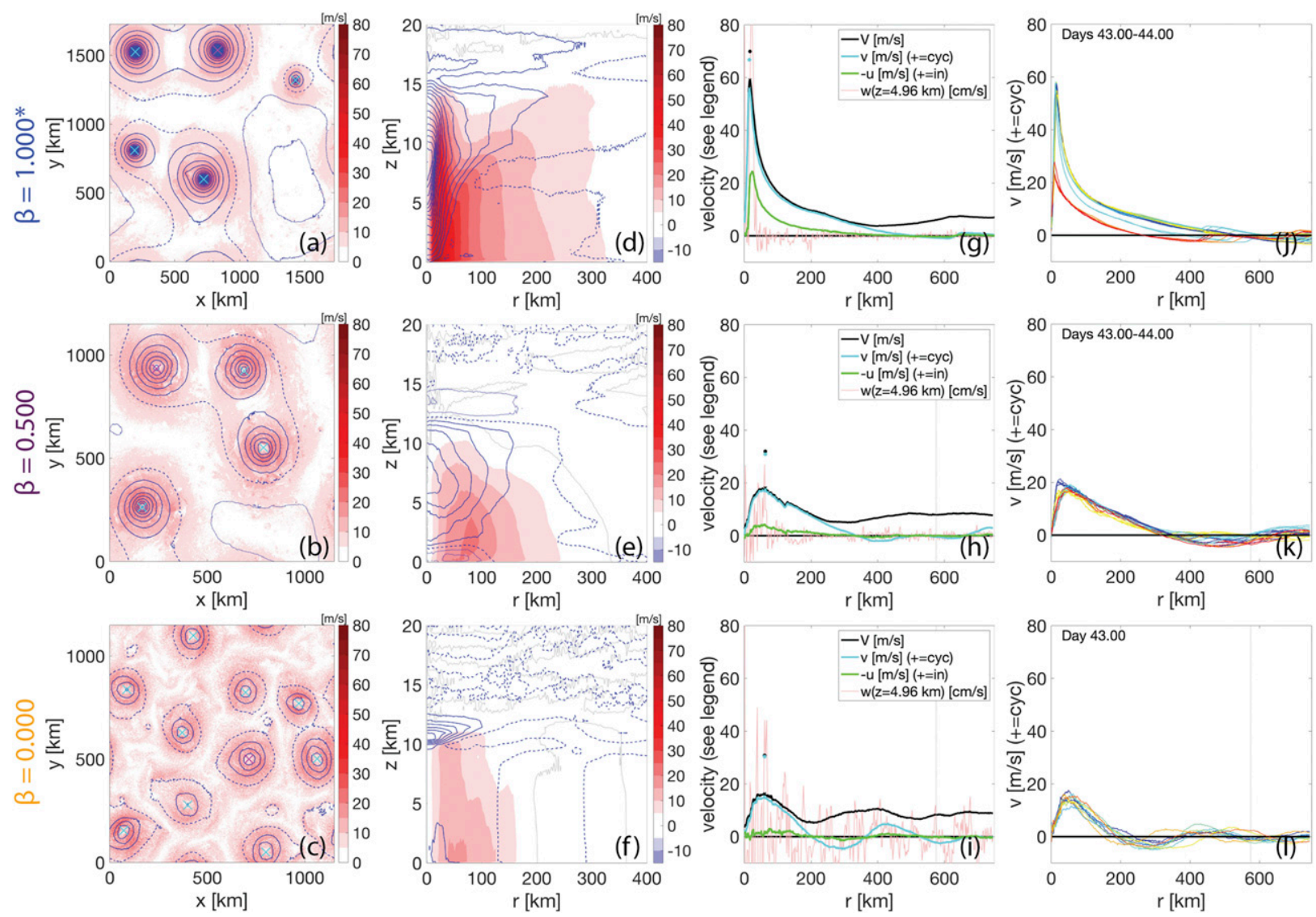

FIG. 4. Snapshots of tropical cyclones on day 43 for (top) moist $\left(\beta=1.000^{*}\right)$, (middle) half-dry $(\beta=0.500)$, and (bottom) dry $(\beta=0.000)$ simulations. (a) $-(\mathrm{c})$ Plan view of wind speed $V$ (colors, $\mathrm{m} \mathrm{s}^{-1}$ ) and negative pressure perturbation $-p^{\prime}(\mathrm{contours}$; interval $=3 \mathrm{hPa}$, dashed $=0)$ at the lowest model level $(z=37.5 \mathrm{~m})$ with storm centers $(\times)$. $(\mathrm{d})-(\mathrm{f})$ Radius-height cross section of tangential wind speed $v$ (colors, $\mathrm{m} \mathrm{s}^{-1}$; red positive, blue negative, gray line $=0$ ) and temperature perturbation from horizontal domain mean $T^{\prime}$ (contours; interval $=1 \mathrm{~K}$, solid positive, dashed $=0$ ) for storms marked with the magenta $\times$ in $(\mathrm{a})-(\mathrm{c})$. (g)-(i) Azimuthal-mean radial profiles of horizontal wind speed $V$ (black line), tangential ( $v$, blue line) and radial ( $-u$, green line) velocity components at $z=37.5 \mathrm{~m}$, and midlevel $(z=4.96 \mathrm{~km})$ vertical velocity $w$ (red line), with maximum $2 \mathrm{D}$ near-surface tangential velocity $v$ (blue dot) and total horizontal wind speed $V$ (black dot) for storms shown in (d)-(f). (j)-(1) Radial profile of tangential wind for all storms in (a)-(c) for five consecutive 6-hourly time steps. Profiles in (l) are shown only for the initial time step for clarity.

simulations show typical characteristics of a tropical cyclone in all cases. These characteristics include an upperlevel warm core at the storm center and a tangential wind speed that peaks near the surface at small finite radius and decays with both radius and height. The dry storm circulation varies weakly with height through the troposphere, and by association (thermal wind balance) its warm core is confined to a relatively thin layer below the tropopause. The moist simulation shows storms with a deeper warm core and a more gradual decay in tangential winds with height. The half-dry simulation lies between the two, but unlike either limiting case has a low-level cold patch coincident with the region of maximum surface winds, likely associated with evaporation of rainfall. The contrasting vertical extent of the warm core seems consistent with the dry simulation having a well-mixed boundary layer that spans the entire depth of the troposphere, in which it is difficult to support strong horizontal temperature gradients.

Though the dry case (Fig. 4f) appears to lack a warm core over much of the depth of the troposphere, its core is still positively buoyant due to its relatively low pressure (i.e., a deep warm core exists on isobaric surfaces). A buoyant core is essential, as it indicates the existence of available potential energy in the system (Yanai 1964) and the production of available potential energy by covariance between heating and buoyancy. To plot the buoyant core, we define a buoyancy temperature $T_{b}$ that is analogous to a density temperature but includes the effects of pressure perturbations at constant $z$. SAM defines the buoyancy $B$ as (Khairoutdinov and Randall 2003, p. 622) 


$$
B=g\left(\frac{T^{\prime}}{\bar{T}}+0.608 q_{v}^{\prime}-q_{n}-q_{p}-\frac{p^{\prime}}{\bar{p}}\right),
$$

where $g$ is the gravitational acceleration, $T$ is temperature, $q_{v}$ is water vapor mixing ratio, $q_{n}$ is nonprecipitating condensate, $q_{p}$ is precipitating condensate, $p$ is pressure, bars denote horizontal means, and primes denote perturbations from the horizontal mean. We can express the buoyancy as

$$
B \equiv g\left(\frac{T_{b}^{\prime}}{\overline{T_{b}}}\right),
$$

where $\overline{T_{b}}$ is the horizontal-mean virtual temperature (for buoyancy SAM assumes $\overline{q_{n}}=\overline{q_{p}}=0$ ), and $T_{b}^{\prime}$ is given by combining Eqs. (7) and (8):

$$
T_{b}^{\prime}=\overline{T_{b}}\left(\frac{T^{\prime}}{\bar{T}}+0.608 q_{v}^{\prime}-q_{n}-q_{p}-\frac{p^{\prime}}{\bar{p}}\right) .
$$

The radius-height structure of $T_{b}^{\prime}$ (Fig. 5) is similar to that for $T^{\prime}$ (Figs. $4 \mathrm{~d}-\mathrm{f}$ ), but the inclusion of pressure perturbations greatly strengthens the buoyant core near the surface and in the lower troposphere. In both the moist and dry limits, $T_{b}^{\prime}$ is positive and nearly uniform with height through the depth of the troposphere. This is not true of the half-dry case, though the vertical distribution of $T_{b}^{\prime}$ is more uniform than that of $T^{\prime}$. In particular, the lowlevel cold anomaly $T^{\prime}$ appears as a warm anomaly in $T_{b}^{\prime}$, indicating that the low-level core of the storm is still positively buoyant but less buoyant than air at mid- to upper levels. Understanding the processes that act to reduce the buoyancy of the low-level core in the half-dry case is beyond the scope of this work, but it could yield insight into why no storms form at low-intermediate $\beta$.

Radial profiles of azimuthally averaged near-surface horizontal winds and midlevel vertical winds for each example storm show low-level inflow at all radii within each cyclone's circulation, and upward motion concentrated near the radius of maximum wind (Figs. $4 \mathrm{~g}-\mathrm{i}$ ). The inflow in each storm is maximized just beyond the radius of maximum wind, with progressively less smoothness for the half-dry and dry cyclones relative to the moist one. In the moist and half-dry storms, convective updrafts are largely confined to a narrow annulus in the inner core of the storm, whereas in the dry storm convective updrafts occur at all radii. All storms exhibit anticyclonic flow beyond some finite outer radius $r_{0}$ (i.e., $v$ decreases below zero), with oscillations in $v$ in the dry case due to sampling the winds from near and far sides of the surrounding ring of cyclones.

Finally, radial profiles of the tangential wind for all identified storms (on day 43) reveal that radial profiles
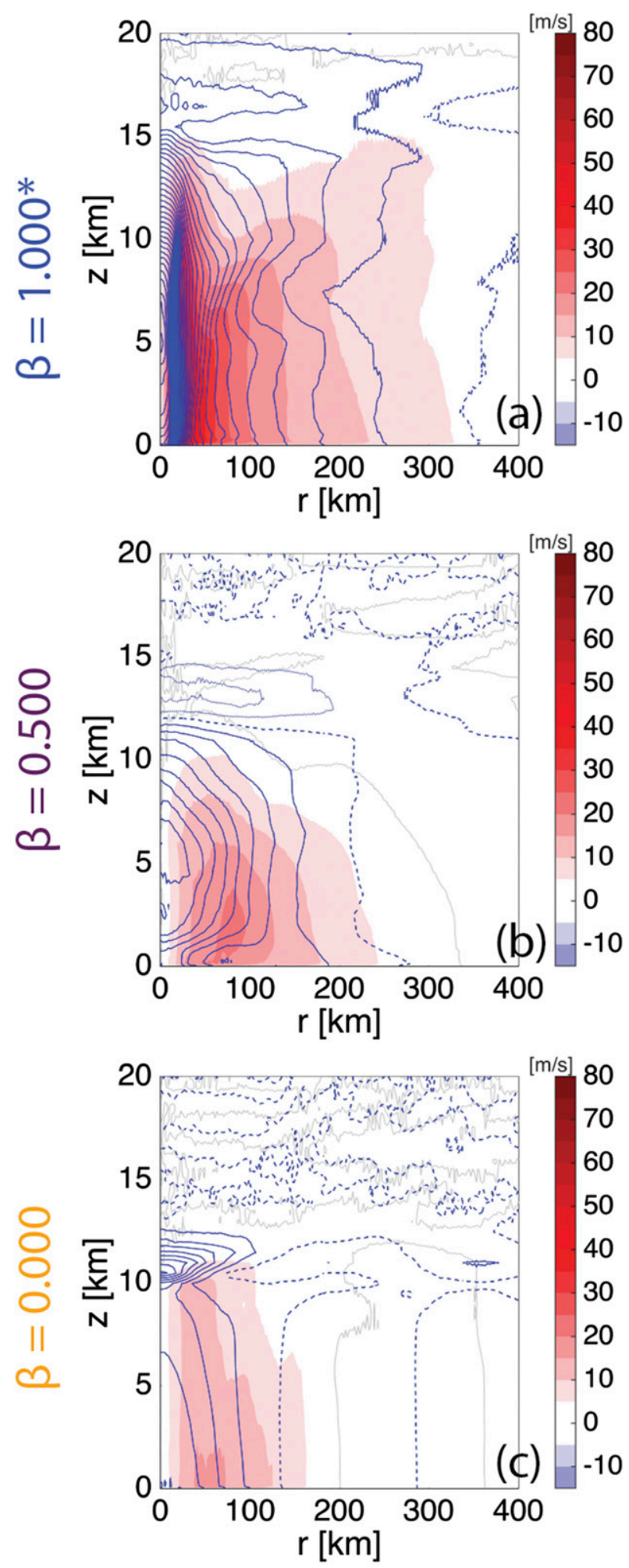

FIG. 5. As in Figs. 4d-f, but for $T_{b}^{\prime}$ in lieu of $T^{\prime}$. 

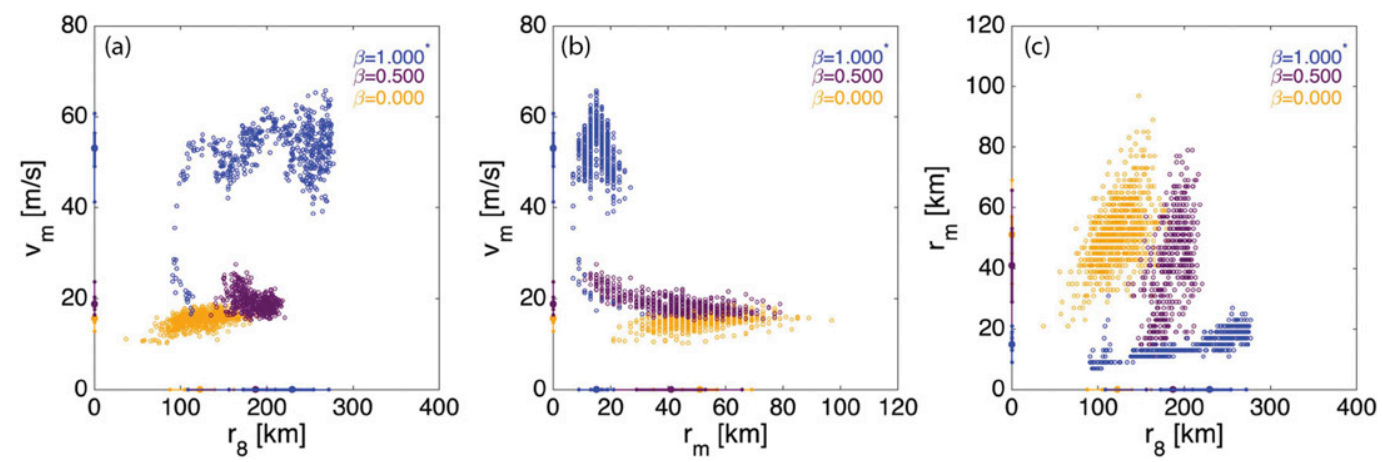

FIG. 6. Statistical covariability among $v_{m}, r_{m}$, and $r_{8}$ across moist $\left(\beta=1.000^{*}\right)$, half-dry $(\beta=0.500)$, and dry $(\beta=0.000)$ simulations for days 40-70: (a) $v_{m}$ and $r_{8}$, (b) $v_{m}$ and $r_{m}$, and (c) $r_{m}$ and $r_{0}$. Median, interquartile range (thick line), and 5th-95th-percentile range (thin line) of individual variables plotted on their respective axes. Storms with $v_{m}<10 \mathrm{~m} \mathrm{~s}^{-1}$ have been excluded.

vary across storms in the moist case (Fig. 4j), but share a common radial structure across storms in the half-dry and dry cases (Figs. 4k,1). This behavior mirrors the wide variance of storm size seen in the real world (Merrill 1984; Chavas et al. 2016), as well as the consistent radial structure of the wind field for a given storm size noted in observations and models and predicted by theory (C15; Chavas and Lin 2016; Reed and Chavas 2015). We speculate that the much slower temporal variation of storm size in the moist case relative to the dry or even half-dry case may be tied to the longer time scales for radiatively induced subsidence in the environment outside of the storm inner cores; this topic is addressed in section $3 \mathrm{f}$.

\section{c. Statistical relationships among moist and dry storm parameters}

We next examine the relationships among structural parameters $\left(r_{8}, r_{m}, v_{m}\right)$ for moist, half-dry, and dry cyclones for simulation days 40-70 (Fig. 6; corresponding statistics in Table 1). We use $r_{8}$ here as a measure of outer storm size because it is less variable in time than $r_{0}$, in line with previous work (Schenkel et al. 2017; Chavas et al. 2017); we discuss $r_{0}$ in subsequent sections. Moist storms are larger and more intense than either half-dry or dry storms, and more variable in both size $r_{8}$ and intensity $v_{m}$ (Fig. 6a). The coefficient of variation for $r_{8}$ is largest in the moist simulation (0.24), reflecting the persistent variability in size across storms displayed in Fig. 4j. This quantity is much smaller for the half-dry simulation (0.09), indicating storms have a tight distribution of sizes, but only slightly below the moist case for the dry simulation (0.19), perhaps indicating an increase in turbulent variability in the dry system. Outer size and intensity show a slight positive correlation for dry storms, but little correlation for half-dry and moist storms, as is found in nature (Merrill 1984; Chavas et al.
2016) and permitted by theory (Chavas and Lin 2016). In association with their greater intensities, moist storms have smaller and less variable radii of maximum winds than either half-dry or dry storms (Fig. 6b). Storms for all three surface wetness values show a positive correlation between radius of maximum winds and outer size, but $r_{m}$ depends more strongly on $r_{8}$ for dry and half-dry simulations (Fig. 6c). Overall, the cluster of half-dry storms in $\left(r_{8}, r_{m}, v_{m}\right)$ space falls between those for moist and dry storms, but lies closer to the dry storm cluster due to the low intensities of dry and half-dry storms.

The joint dependences of the radius of maximum tangential wind $r_{m}$ and the central pressure deficit, $\Delta p=p_{c}-\bar{p}$, on outer storm size and intensity $\left(r_{8}\right.$ and $\left.v_{m}\right)$ differ slightly between moist, half-dry, and dry cyclones (Fig. 7). Each value of surface moisture gives rise to storms that occupy a distinct region of $\left(r_{8}, v_{m}\right)$ space, but in all three simulations, $r_{m}$ is greatest for large and weak storms in accordance with theory (Emanuel 1986; Emanuel and Rotunno 2011; Chavas and Lin 2016). Large dry storms show only a weak anticorrelation between intensity and $r_{m}$, unlike moist and half-dry storms (Figs. 7a,c,e). The central pressure deficit is larger for storms with higher maximum wind speeds and larger size (Figs. 7b,d,f), in accordance with recent work (Chavas et al. 2017).

\section{d. Rotating RCE from moist to dry limits}

Although cyclones form readily in both moist and dry limits, spontaneous genesis is strikingly absent for intermediate values of surface wetness, from $\beta=0.01-0.3$ (Fig. 8). All simulations that form cyclones $(\beta=0-0.003$ and $\beta=0.4-1^{*}$ ) have surface kinetic energy dissipation $\left(\rho c_{D} \overline{V^{3}}\right)$ values of $1-4 \mathrm{~W} \mathrm{~m}^{-2}$ (though up to $10 \mathrm{~W} \mathrm{~m}^{-2}$ immediately following genesis for moist storms), mean surface wind speeds of about $8 \mathrm{~m} \mathrm{~s}^{-1}$, and maximum surface wind speeds of $35-75 \mathrm{~m} \mathrm{~s}^{-1}$. In between these 
TABLE 1. Statistics of quantities for each simulation displayed in Fig. 6, including the median, first quartile (25\%), third quartile (75\%), mean $\mu$, standard deviation $\sigma$, and coefficient of variation $c_{v}$.

\begin{tabular}{|c|c|c|c|c|c|c|c|}
\hline Simulation & Quantity & Median & $25 \%$ & $75 \%$ & $\mu$ & $\sigma$ & $c_{v}$ \\
\hline \multirow[t]{5}{*}{$\beta=1.000^{*}$} & $v_{m}\left(\mathrm{~m} \mathrm{~s}^{-1}\right)$ & 53.1 & 49.0 & 56.4 & 51.8 & 7.7 & 0.15 \\
\hline & $\Delta P(\mathrm{hPa})$ & 74.8 & 65.8 & 81.5 & 72.2 & 15.0 & 0.21 \\
\hline & $r_{m}(\mathrm{~km})$ & 15.0 & 13.0 & 19.0 & 15.3 & 3.8 & 0.25 \\
\hline & $r_{8}(\mathrm{~km})$ & 229.3 & 173.1 & 254.1 & 211.9 & 51.7 & 0.24 \\
\hline & $r_{0}(\mathrm{~km})$ & 476.4 & 383.9 & 512.4 & 469.9 & 108.6 & 0.23 \\
\hline \multirow[t]{5}{*}{$\beta=0.500$} & $v_{m}\left(\mathrm{~ms}^{-1}\right)$ & 18.9 & 17.7 & 20.4 & 19.3 & 2.2 & 0.11 \\
\hline & $\Delta P(\mathrm{hPa})$ & 20.4 & 18.7 & 22.5 & 20.8 & 2.7 & 0.13 \\
\hline & $r_{m}(\mathrm{~km})$ & 41.0 & 29.0 & 53.0 & 40.9 & 15.3 & 0.37 \\
\hline & $r_{8}(\mathrm{~km})$ & 186.9 & 172.4 & 199.8 & 185.3 & 17.0 & 0.09 \\
\hline & $r_{0}(\mathrm{~km})$ & 329.9 & 313.6 & 343.8 & 331.6 & 29.4 & 0.09 \\
\hline \multirow[t]{5}{*}{$\beta=0.000$} & $v_{m}\left(\mathrm{~m} \mathrm{~s}^{-1}\right)$ & 15.7 & 14.8 & 16.4 & 15.5 & 1.4 & 0.09 \\
\hline & $\Delta P(\mathrm{hPa})$ & 10.1 & 8.4 & 11.7 & 10.1 & 2.2 & 0.21 \\
\hline & $r_{m}(\mathrm{~km})$ & 51.0 & 45.0 & 57.0 & 51.4 & 10.6 & 0.21 \\
\hline & $r_{8}(\mathrm{~km})$ & 122.2 & 104.9 & 139.4 & 122.7 & 23.3 & 0.19 \\
\hline & $r_{0}(\mathrm{~km})$ & 226.1 & 198.3 & 263.1 & 241.2 & 67.0 & 0.28 \\
\hline
\end{tabular}

two clusters of simulations with dry and moist storms is a "no-storms-land" where spontaneous genesis does not occur, marked by smaller surface kinetic energy dissipation of $0.2-0.5 \mathrm{~W} \mathrm{~m}^{-2}$, average surface wind speeds of less than $5 \mathrm{~m} \mathrm{~s}^{-1}$, and maximum surface wind speeds of $20-30 \mathrm{~m} \mathrm{~s}^{-1}$.

It would be tempting to conclude that this no-stormsland acts as a divider between moist $\left(\beta=0.4-1^{*}\right)$ and dry
( $\beta=0-0.003$ ) blocs of storms. But based on the analysis above, and the vortex statistics described here, this conclusion would be misleading: the storms with $\beta=0.4-0.6$ are truly intermediate states between the fully moist and dry limits, sharing features of both. The $\beta=0.4$ and $\beta=0.5$ cases have median tangential winds in tracked cyclones of $15-20 \mathrm{~m} \mathrm{~s}^{-1}$ - similar to the fully dry and the nearly dry storms with $\beta=0.001$ and $\beta=0.003$-yet the
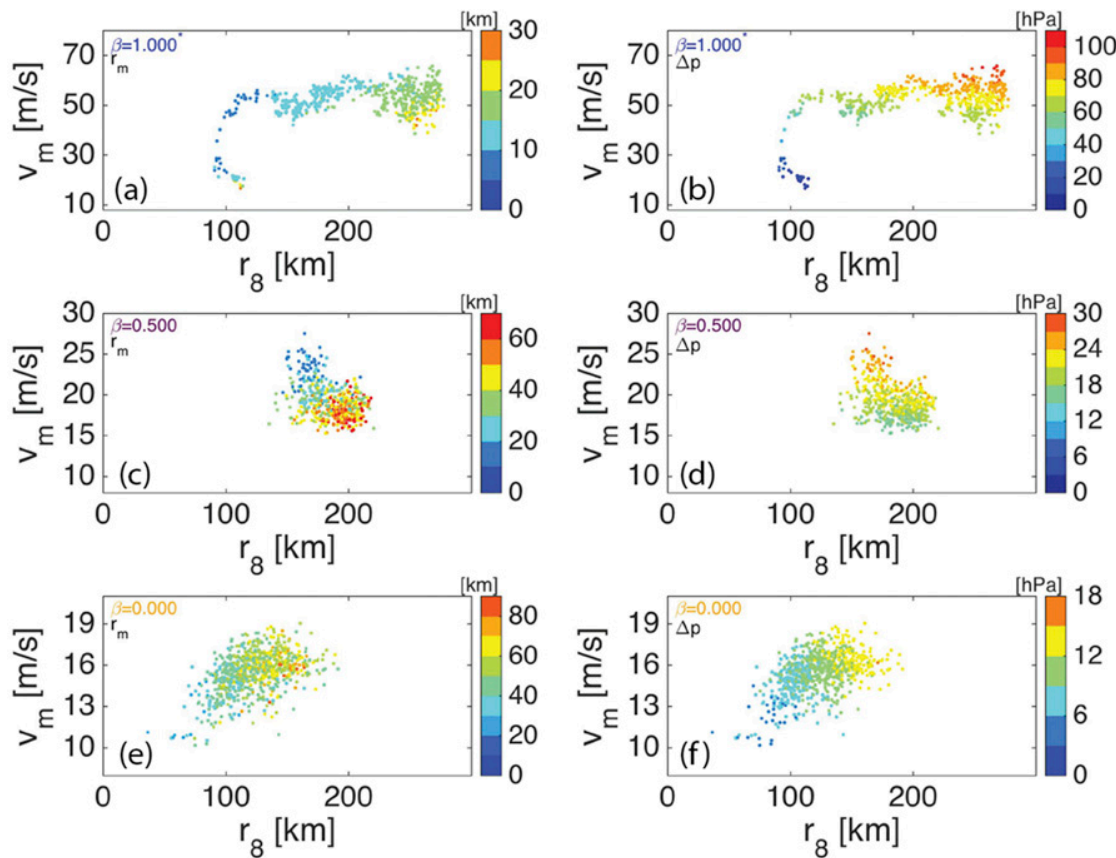

FIG. 7. Joint variability of cyclone structure parameters for days 40-70. (a) $r_{m}$ as function of $v_{m}$ and $r_{8}$ for the moist simulation $\left(\beta=1.000^{*}\right)$. (b) As in (a), but for the central pressure deficit. (c),(d) As in (a) and (b), but for the half-dry simulation $(\beta=0.500)$. (e),(f) As in (a) and (b), but for the dry simulation $(\beta=0.000)$. Note that color scales differ for each panel and that storms with $v_{m}<10 \mathrm{~m} \mathrm{~s}^{-1}$ have been excluded. 


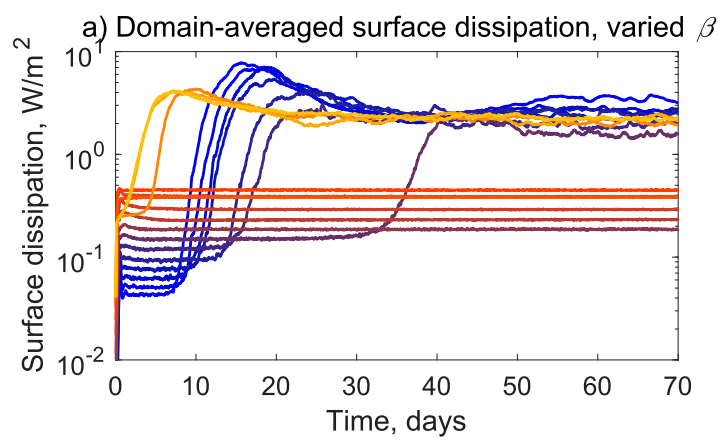

c) Domain-averaged surface dissipation, varied $T_{S}$

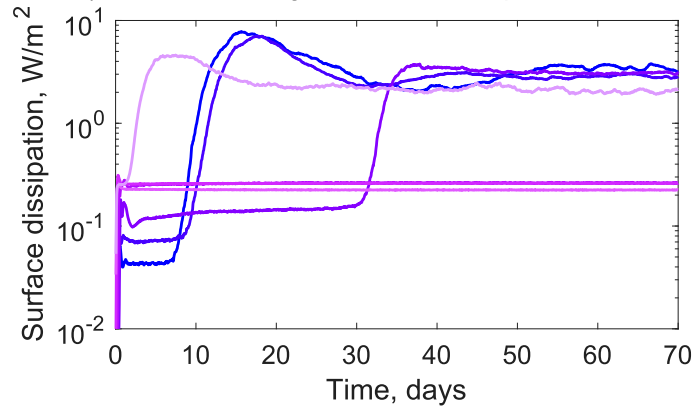

b) Surface wind statistics, varied $\beta$
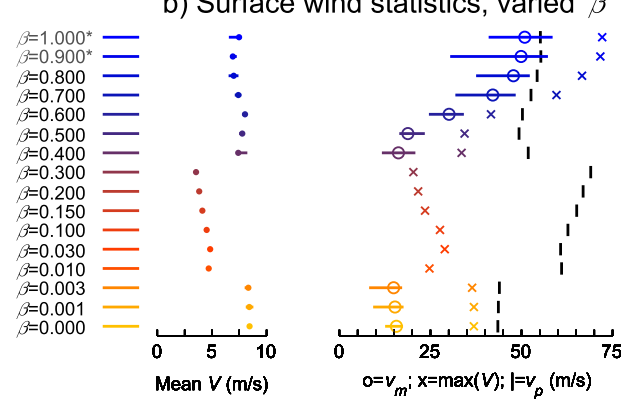

d) Surface wind statistics, varied $T_{S}$

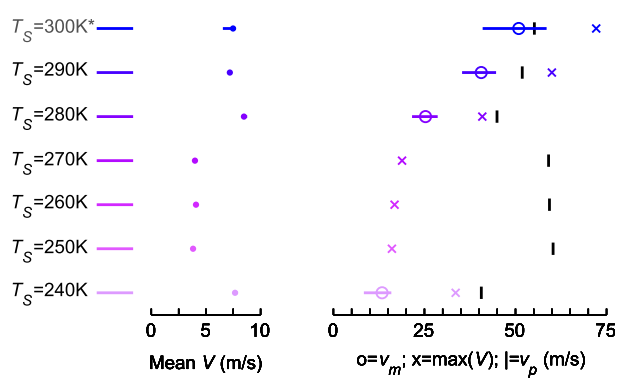

FIG. 8. (left) Time series of domain-averaged surface kinetic energy dissipation for (a) simulations with varied surface wetness $\beta$ and (c) simulations with varied surface temperature $T_{S}$ (note the logarithmic vertical axes). (right) Median values over time from days 40-70 are shown for the domain-averaged surface wind speed $\bar{V}$ (dots), maximum tangential winds associated with tracked cyclones $v_{m}$ (circles), and domain-maximum surface wind speeds $\max (V)(\times)$ with (b) varied $\beta$ and (d) varied $T_{S}$. Horizontal lines in (b) and (d) around markers for domainmean and maximum cyclone azimuthal winds indicate the 5th-95th percentiles of each value (across time for the domain-mean wind and across time and cyclones for the azimuthal winds). Vertical black lines in (b) and (d) indicate potential intensity $v_{p}$ averaged from days 40-70 of each simulation and calculated from the domainaverage surface enthalpy disequilibrium [Eq. (5)].

convective dynamics and ambient stratification in these simulations remain fundamentally moist. These storms are also intermediate in structure between the moist and dry limits, with large radii of maximum winds as compared to the very moist storms from $\beta=0.8-1^{*}$, and more eyewall asymmetry than the moist storms, but less than the dry ones (Figs. 1a,c,e).

Potential intensity $v_{p}$, shown by black vertical lines in Fig. $8 \mathrm{~b}$, acts as a strong upper bound for $v_{m}$ of moist cyclones, but semidry and dry cyclones have much lower values of $v_{m}$ relative to $v_{p}$. Note that the absolute maximum of $V$ in the domain exceeds $v_{p}$ for moist storms, because potential intensity theory does not include azimuthal asymmetries or small-scale convective gusts. In simulations with $\beta=0-0.003$, the combination of convective gusts and asymmetric eyewall structure leads to maximum surface wind speeds that are roughly twice $\boldsymbol{v}_{m}$. The upward jump in potential intensity in no-stormsland arises from the constraint in RCE that surface enthalpy disequilibrium vary inversely with the domainaverage wind speed, for fixed column-integrated radiative cooling. Thus, the weaker mean winds that occur for simulations without any cyclones allow for greater surface enthalpy disequilibrium and potentially stronger storms, but if storms were to form, the average surface wind speed would increase and the potential intensity would fall. This negative feedback between cyclone activity and global $v_{p}$ also causes surface dissipation to overshoot its equilibrium value before stabilizing in simulations that experience cyclogenesis (Fig. 8), and is likely relevant for predicting the properties of cyclones in a turbulent TC-world from the initial sounding.

Simulations across a range of surface temperatures show a similar transition from moist to dry as the surface is cooled (Figs. 8c,d). Simulations that form cyclones $\left(T_{S}=240\right.$ and $\left.T_{S}=280-300 \mathrm{~K}\right)$ show kinetic energy dissipation and wind speeds close to those from the varied- $\beta$ simulations (note that the $T_{S}=300 \mathrm{~K}^{*}$ simulation with $\beta=1^{*}$ is shared between these groups). Genesis is delayed and maximum wind speeds weaken as $T_{S}$ is reduced from $300 \mathrm{~K}^{*}$ to $280 \mathrm{~K}$, akin to the effects of reduced $\beta$ from $1^{*}$ to 0.4 . A no-storms-land is found for simulations with $T_{S}$ from 250 to $270 \mathrm{~K}$, with slightly weaker convective turbulence than the semidry simulations 
that lack cyclones. One simulation with $T_{S}=240 \mathrm{~K}$ parallels the set of dry TC-world simulations from $\beta=0-0.003$, showing near-immediate genesis, surface kinetic energy dissipation $\sim 2 \mathrm{~W} \mathrm{~m}^{-2}$, average surface wind speeds of $\sim 8 \mathrm{~m} \mathrm{~s}^{-1}$, and maximum surface wind speeds $\sim 35 \mathrm{~m} \mathrm{~s}^{-1}$. The $T_{S}=280-\mathrm{K}$ simulation strongly resembles the $\beta=0.400$ simulation in terms of the delayed genesis of cyclones (Figs. 8a,c), suggesting a similar process gradually impedes cyclogenesis as the surface is dried or cooled, but this similarity may be a coincidence.

As found by Zhou et al. (2014), as well as Chavas and Emanuel (2014) in axisymmetric geometry, the outer size of our simulated cyclones does not appear to scale cleanly with either the deformation radius $N H / f$ or a potential intensity radius $v_{p} / f$ (Fig. 9). The radius of vanishing azimuthal winds $r_{0}$ decreases by about a factor of two going from moist to dry or warm to cold limits, but $N H / f$ decreases by a factor of roughly six, whereas $v_{p} / f$ decreases by only $30 \%-40 \%$. Instead, $v_{p} / f$ appears to more closely follow the convective vortex size $r_{C}$, which varies only weakly as surface moisture or temperature is decreased; $r_{C}$ shows a hint of smaller values for intermediate $\beta$ or $T_{S}$ than for either warm/moist or dry/cold storms. Moreover, the convective vortex size appears approximately bounded from above by $v_{p} / f$, as suggested by Emanuel (2004), with dry storms bumping up more closely against this upper bound, suggesting that the intensities of dry storms might be limited by the storms becoming too large. Thus, our results indicate that $v_{p} / f$ is a more relevant length scale for the inner convective core of a tropical cyclone, while the overall size of the storm may further depend on a length scale associated with the structure of the nonconvecting outer circulation, in line with the axisymmetric results of Chavas and Emanuel (2014). The circulation structure is analyzed in section $3 \mathrm{f}$.

Cyclone properties in both sets of simulations can also be compared using nondimensional intensity $v_{m} / v_{p}$ and the inner-core Rossby number Ro $=v_{m} / f r_{m}$ (Fig. 10). Median $v_{m} / v_{p}$ lies between 0.75 and 1 for surfaces with $\beta=0.7-1^{*}$ and with $T_{S}=290 \mathrm{~K}$ to $300 \mathrm{~K}^{*}$, but decreases rapidly with further surface drying to about 0.3 for $\beta=0.4-$ with similar values for the nearly dry and fully dry $\beta=0-0.003$ simulations (Figs. 10a,c). Although increased asymmetry and convective gustiness in the dry limit makes $v_{m} / v_{p}$ an imperfect measure of intensity, Fig. 8 shows that $V / v_{p}$ also decreases with surface drying (albeit nonmonotonically for varied $\beta$ ). We note as an aside that our $1152 \mathrm{~km} \times 1152 \mathrm{~km}$ simulation with a fully moist surface $(\beta=1)$, which eventually collapsed to one storm, showed much smaller normalized median intensity of $v_{m} / v_{p}=0.7$ and much greater variability in time a) Outer size distributions, varied $\beta$

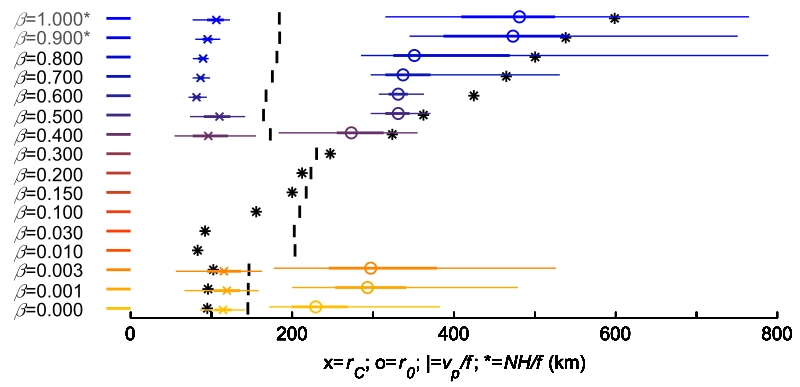

b) Outer size distributions, varied $T_{S}$

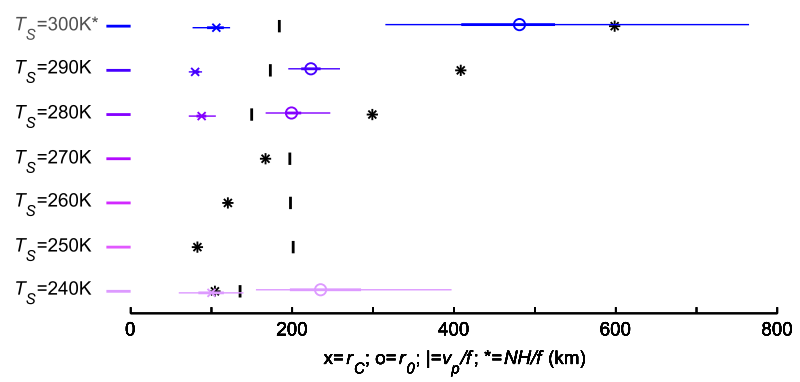

FIG. 9. Metrics of storm size, for simulations with (a) varied surface moisture and (b) varied surface temperature. The medians for all cyclones from days 40-70 of fully convective vortex extent $r_{C}$ [Eq. (6)] are shown with $\times$ symbols, and the larger radius of vanishing winds $r_{0}$ with open circles. Thin horizontal lines indicate 5th-95th percentiles of each value, and thicker lines indicate interquartile range. For each simulation, vertical black bars denote the length scale given by the time-average of $v_{p} / f$ [Eq. (5) defines the simplified potential intensity], and asterisks denote the deformation radius $N H / f$, where $N$ is the density-weighted buoyancy frequency of the troposphere and $H$ is the tropopause height (defined as the height of the last model level where the lapse rate exceeds $2 \mathrm{~K} \mathrm{~km}^{-1}$ ), both taken from a time-mean domain-average temperature profile.

(5th-95th percentiles of $v_{m} / v_{p}$ ranging from 0.38 to 1.06 ), consistent with Zhou et al. (2014), who found that cyclone intensity varies intermittently in time in lattice TC-worlds with only one storm. The inner-core Rossby number, $\mathrm{Ro}=v_{m} / f r_{m}$, shows a similar gradual transition between cyclostrophic vortices with Ro $\gg 1$ for warm/ moist surfaces, and gradient-balance vortices with Ro $\sim 1$ for dry/cold surfaces (Figs. 10b,d). Overall, Fig. 10 reinforces our impression that the no-storms-land is not a divider between regimes of moist and dry cyclones, but rather serves to interrupt a gradual transition from moist to dry vortex dynamics.

\section{e. Transient variations in surface properties}

To test whether the no-storms-land can destroy established storms, or merely prevents spontaneous genesis, we conducted four additional simulations where the surface wetness or temperature were ramped up or down in time from initial values that allow spontaneous genesis. 

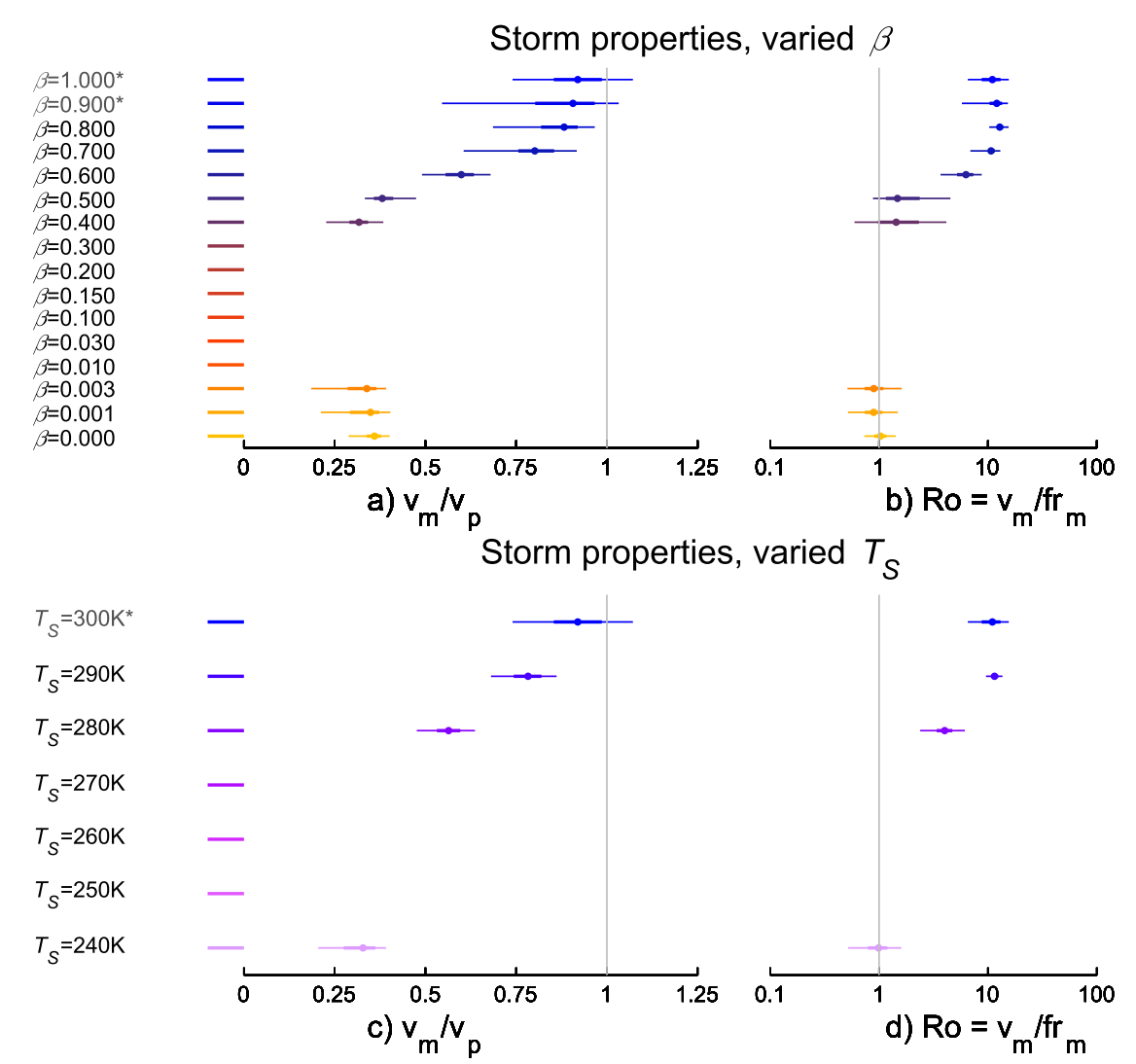

FIG. 10. (a),(c) Dimensionless cyclone intensity $v_{m} / v_{p}$ and (b),(d) inner-core Rossby number Ro $=v_{m} / f r_{m}$. Dots indicate median values for all tracked cyclones from days 40-70, thin horizontal lines indicate 5th-95th percentiles, and thick horizontal lines indicate interquartile range (25th-75th percentiles). (a), (b) Simulations with varied $\beta$ and (c),(d) simulations with varied $T_{S}$. Thin vertical gray lines indicate values of unity for each dimensionless parameter.

We refer to these as " $\beta$-sweep" and " $T_{S}$-sweep" simulations. For the downward $\beta$-sweep simulation we used $\beta(t)=\exp ^{-0.03454 t}$ and for the upward $\beta$-sweep simulation we used $\beta(t)=0.001 \mathrm{exp}^{0.03454 t}$ (with $t$ as time in days), giving transitions between $\beta=1$ and $\beta=0.001$ over each 200-day simulation. For the downward $T_{S}$-sweep simulation we used $T_{S}(t)=300-0.25 t$ and for the upward $T_{S}$-sweep simulation we used $T_{S}(t)=240+0.25 t$; these simulations were run for 240 days each. In the $T_{S}$-sweep simulations, two additional modifications were made in an attempt to limit variations in $v_{p}$ over the course of each simulation and across the two simulations. First, the tropopause temperature was increased or decreased in time at 0.7 times the rate of the surface temperature [i.e., $T_{T}(t)=210-0.175 t$ and $T_{T}(t)=168+0.175 t$ ], maintaining a similar thermodynamic efficiency and tropospheric mass across surface temperatures. Second, the radiative cooling rate relative to the surface temperature was held fixed at $1 \mathrm{~K} \mathrm{day}^{-1}$ (i.e., modifying $\dot{Q}$ to $0.75 \mathrm{~K} \mathrm{day}^{-1}$ in the upward sweep and to $1.25 \mathrm{~K} \mathrm{day}^{-1}$ in the downward sweep). This choice was made because a surface that warms in time will have a greater enthalpy flux than a surface of constant temperature or one that cools in time; varying $\dot{Q}$ is an attempt to offset this.

In all of the four temperature and moisture sweep simulations, cyclones form in moist/warm or dry/cold limits before the boundary conditions transit through no-storms-land, and partially disrupted cyclones persist for tens of days as the simulation passes through the nostorms-land. Therefore, we conclude that the regions of parameter space from $\beta=0.01-0.3$ and $T_{S}=250-270 \mathrm{~K}$ are barriers to spontaneous cyclogenesis, but not to the existence of cyclones.

To present simulations across both $\beta$ and $T_{S}$ on the same axes, we examine whether the surface kinetic energy dissipation $\rho c_{D} \overline{V^{3}}$ normalized by the surface enthalpy flux $F_{k}=E+H$, depends similarly on the surface latent heat flux fraction, $E / F_{k}$, across both suites of simulations. The normalized dissipation $\rho c_{D} \overline{V^{3}} / F_{k}$ is a measure of the global conversion efficiency of convective heat transport into surface frictional dissipation, whereas $E / F_{k}$ is a dimensionless measure of surface 


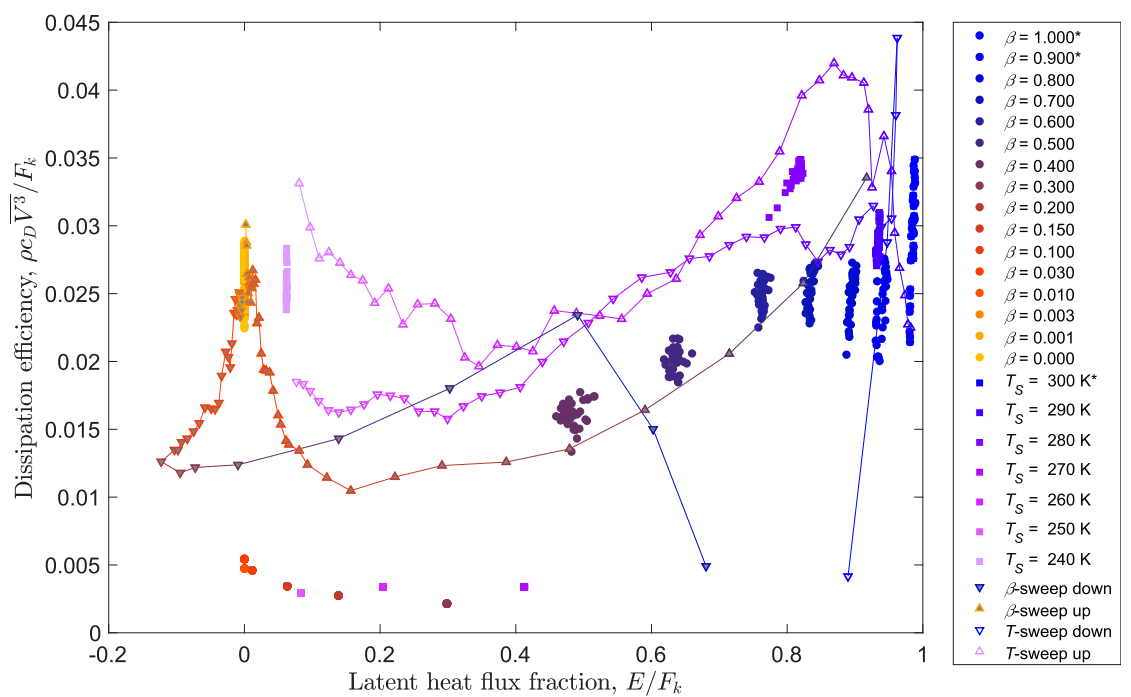

FIG. 11. Plot of domain-averaged normalized surface dissipation, $\rho c_{D} \overline{V^{3}} / F_{k}$, against the fraction of surface enthalpy flux carried by evaporation, $E / F_{k}$, for the two main sets of simulations with varied values of $\beta$ (filled circles) and $T_{S}$ (filled squares), as well as simulations where $\beta$ or $T_{S}$ is swept upward (upward-pointing triangles) or downward (downward-pointing triangles) in time. For the main sets of simulations, daily averages are shown for days 40-70; for the $\beta$-sweep and $T_{S}$-sweep simulations, 5-day averages are shown from day 10 onward.

evaporation, and should tend to 0 in both cold and dry limits. In this nondimensional parameter space, the nostorms-land appears as a common region from $E / F_{k}$ of about $0.1-0.4$, and $\rho c_{D} \overline{V^{3}} / F_{k}<0.005$ (Fig. 11), and dry to nearly dry TC-worlds are disjoint from semidry and moist TC-worlds. All four of the sweep simulations, however, connect the dry and moist regions, and can be clearly distinguished from simulations without cyclogenesis because they maintain dissipation efficiencies greater than 0.01 after cyclones have initially formed. Note that the $\beta$-sweep and $T_{S}$-sweep simulations show considerable spread in dissipation efficiency at any given value of $E / F_{k}$, except in the dry limit. The downward $\beta$-sweep simulation is an oddity because of its numerous points with $E / F_{k}<0$; this occurs because Eq. (3) allows a moist atmosphere to transfer latent heat into the surface if $\beta q_{S}^{*}<q_{1}$, and $\beta$ decreases rapidly enough in time that this condition is satisfied for much of the simulation. Cyclones generally have a different ratio of $E / F_{k}$ than the domain as a whole; for example, cyclones from simulations with $\beta=0.4$ and $\beta=0.5$ have negative latent heat fluxes but very large positive sensible heat fluxes near their eyewalls. Whether the cyclone-scale ratio of evaporation to total enthalpy flux might be used to characterize the transition from moist to dry vortex dynamics is a question left for future work.

\section{f. Vortex structure}

As a final aspect of our analysis, we examine the behavior of the radial structure of the near-surface wind field with varying surface wetness and surface temperature. Figure 12 shows the nondimensional tangential wind profile, $v(r) / v_{p}$, as a function of nondimensional radius $r /\left(v_{p} / f\right)$ for the composite cyclone calculated using the 94 th- 95 th percentile in $v_{m}$ for each simulation, where $v_{p}$ is defined simply as the mean value from Eq. (5) for days 40-70. Dimensional results for composite structure are not qualitatively different, as Fig. 8 shows that $v_{p}$ varies by only $\sim 20 \%$ for simulations with vortices, and $f$ is fixed. We choose this range of high percentiles to select a small number of relatively intense storms within each simulation; the narrow range minimizes nonphysical results that can arise with averaging, as there is no single length scale applicable to the entire vortex (Chavas and Lin 2016).

As either $\beta$ or $T_{S}$ is decreased, $v_{m} / v_{p}$ decreases rapidly (as in Fig. 10). This reduction in intensity is associated with both an outward expansion of the inner circulation, including a gradual increase in nondimensional radius of maximum wind, and an inward contraction of the outer circulation, such that the ratio of inner to outer storm size, $r_{m} / r_{0}$, increases monotonically with surface drying or cooling. An increase in $r_{m}$ with decreasing $v_{m}$ is expected from angular momentum considerations (Emanuel 1986) and is commonly observed in nature (Stern et al. 2015). A larger shift occurs for the dry/cold limit, in which the inner circulation jumps outward and the outer tail of the wind profile decays rapidly to zero. The composite wind profiles for the $\beta=0$ and $T_{S}=240-\mathrm{K}$ 
a) Composite structure for high-intensity cyclones over $\beta$

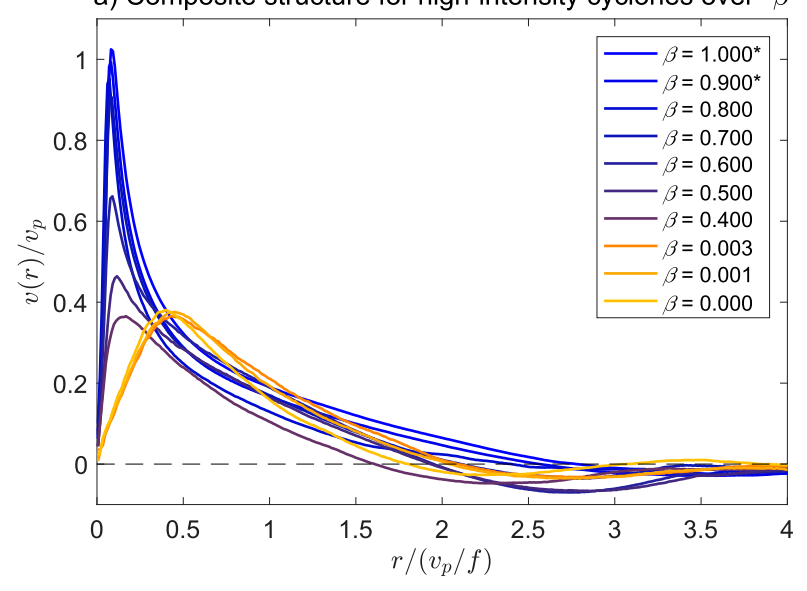

b) Composite structure for high-intensity cyclones over $\mathrm{T}_{\mathrm{S}}$

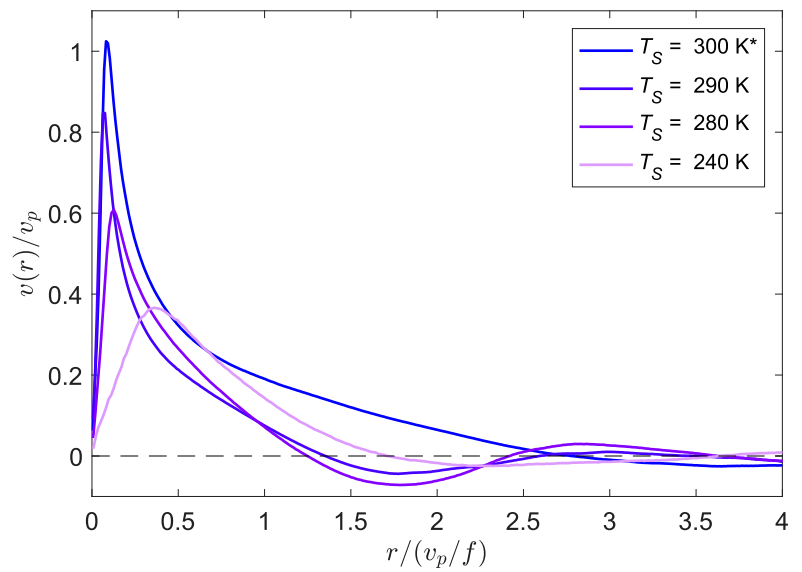

FIG. 12. Nondimensional tangential wind profiles $v(r) / v_{p}$ plotted as a function of nondimensional radius $r /\left(v_{p} / f\right)$ for composites of cyclones from the 94th-95th percentile of tangential wind speeds for (a) simulations across different surface wetness and (b) simulations across different surface temperatures. For each simulation, the mean potential intensity [Eq. (5)] from days $40-70$ is used to scale both intensity and radius.

simulations are nearly identical, indicating strong dynamical similarity between varying surface wetness and temperature.

We seek to understand this behavior by examining our moist, half-dry, and dry storms using the wind structure theory of C15. C15 provided a two-region physical model for the radial structure of the low-level tangential wind, merging the solution of Emanuel and Rotunno (2011) for the inner ascending region with the solution of Emanuel (2004) for the outer descending region. The inner solution assumes that small-scale turbulence sets the entropy stratification of the outflow and thus, for a slantwise-neutral vortex, sets the radial gradient of entropy and angular momentum at the top of the boundary layer in the ascent region of the storm. The outer solution assumes a slab boundary layer in gradient Ekman balance and whose Ekman suction rate equals the radiativesubsidence rate $w_{\text {cool }}$ of air overlying the boundary layer. The $\mathrm{C} 15$ model is specified with two storm-specific parameters - the maximum wind speed and any single measure of storm size - as well as three environmental parameters: the Coriolis parameter at the center of the storm $f$, the ratio of the exchange coefficients of enthalpy and momentum $c_{K} / c_{D}$, and the ratio of the freetropospheric radiative-subsidence rate to the drag coefficient $w_{\text {cool }} / c_{D}$ outside the convective activity of the storm. This model captures the radial structure of winds in storms from both observations (C15) and GCM simulations (Reed and Chavas 2015). In our simulations, $c_{K}=c_{D}=1.5 \times 10^{-3}$, and $f=3 \times 10^{-4} \mathrm{~s}^{-1}$ are all fixed. We define $w_{\text {cool }}$ as the mean vertical velocity within the layer $z \in[2,5] \mathrm{km}$ for all grid points where $p_{s}>\overline{p_{s}}$ and $V<5 \mathrm{~m} \mathrm{~s}^{-1}$ for days 40-70. This definition corresponds to the mean lower-tropospheric vertical velocity in the environment largely between the storms, following the methodology of Reed and Chavas (2015). The resulting radiative-subsidence rates are $0.16 \mathrm{~cm} \mathrm{~s}^{-1}$ (interquartile range of daily values: $[0.11,0.20])$ for the moist storm, $0.35 \mathrm{~cm} \mathrm{~s}^{-1}([0.24,0.46])$ for the half-dry storm, and $5.98 \mathrm{~cm} \mathrm{~s}^{-1}$ ([5.33, 6.66]) for the dry storm. The increase in $w_{\text {cool }}$ as the atmosphere dries reflects a decrease in lowertropospheric static stability and associated decrease in the asymmetry between updrafts and downdrafts that is fundamental to moist convection (Bjerknes 1938).

Using $r_{8}$ as the metric of storm size, the $\mathrm{C} 15$ model successfully captures the gross structure of each storm (from Figs. 4g,h,i) and fits the wind profile reasonably well beyond $r_{m}$, particularly for the moist storm (Fig. 13a). Moreover, the theory anticipates the decreased size of the outer wind field for the half-dry and dry storms. This behavior arises because $w_{\text {cool }}$ increases moving from the moist to dry limit; larger values of $w_{\text {cool }}$ equate to a larger Ekman suction rate and thus largermagnitude negative vorticity, or a wind profile that decays more rapidly with radius. To further demonstrate this effect, Fig. 13b displays the $\mathrm{C} 15$ solution for radiativesubsidence rates spanning $0.2-20 \mathrm{~cm} \mathrm{~s}^{-1}$ with all other parameters held fixed. As $w_{\text {cool }}$ is increased, the outer solution wind profile decays more rapidly toward zero. In the limit of large $w_{\text {cool }} / c_{D}$, the outer and inner solutions of $\mathrm{C} 15$ converge in the outer storm circulation. This result may be interpreted in two ways. Physically, dry storms may exhibit persistent convection at all radii and thus no transition to a nonconvecting region exists. Mathematically, in the large $w_{\text {cool }}$ limit, the outer solution of C15 approaches constant angular momentum at large radii, which is identical to the inner solution. The C15 model thus predicts a wind profile that converges 

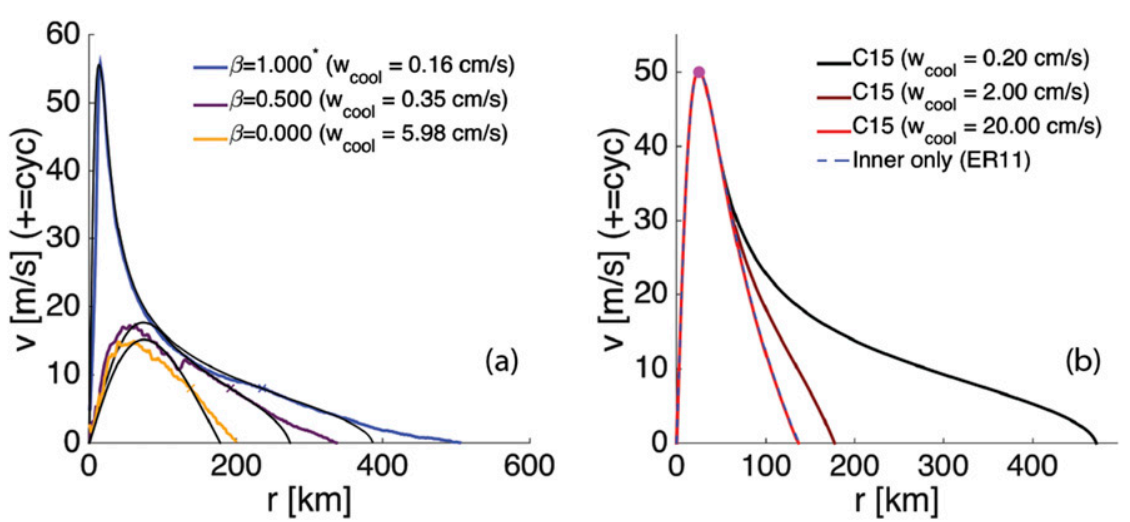

FIG. 13. (a) Fit of $\mathrm{C} 15$ solution (black curves) to example tangential wind profiles from experiments $\beta=1.000^{*}, \beta=0.500$, and $\beta=0.000$ (shown in Figs. $4 \mathrm{~g}-\mathrm{i}$ ). Model is fit to $v_{m}$ and $r_{8}$ $(\times)$, with parameter values matching the simulations: $c_{K} / c_{D}=1, c_{D}=1.5 \times 10^{-3}$, and $w_{\text {cool }}$ (see legend) calculated as the mean vertical velocity within the layer $z \in[2,5] \mathrm{km}$ for all grid points where $p_{s}>\overline{p_{s}}$ and $V<5 \mathrm{~m} \mathrm{~s}^{-1}$ for the days 40-70. (b) C15 solution for a range of values of $w_{\text {cool }}$, along with the corresponding the inner-core-only solution of Emanuel and Rotunno (2011); parameter values are: $v_{m}=50 \mathrm{~m} \mathrm{~s}^{-1}, r_{m}=25 \mathrm{~km}, f=3 \times 10^{-4} \mathrm{~s}^{-1}$, and $c_{K}=c_{D}=1.5 \times 10^{-3}$.

purely to the inner solution alone as one approaches the dry limit, in line with the results of our simulations. One notable discrepancy occurs at the far outer edge of the storm where the simulated profile extends to larger radii than predicted by the outer solution, which is likely due to the assumption that $V \approx v$ in the surface stress; this assumption breaks down as $v \rightarrow 0$ (Figs. $4 \mathrm{~g}-\mathrm{i}$ ).

Overall, these results for outer circulation structure, in conjunction with a $v_{p} / f$ length scale for the fully convecting vortex size, explain why outer storm size does not cleanly follow any single length scale: outer size integrates the two independent length scales associated with the inner convecting and outer nonconvecting circulations. This conclusion is similar to that found in Chavas and Emanuel (2014) and is intrinsic to the C15 model, but still leaves room for further work on the analytic dependencies of the outer circulation scale and the fraction of the storm that is convecting.

\section{Conclusions}

We have found that dry tropical cyclones readily form and persist in rotating 3D RCE, and that the dry limit does not appear to be singular: the moist-dry transition is marked by a gradual change in vortex properties, including structure and intensity. Dry and semidry storms are weaker in both absolute terms and relative to their potential intensities than standard fully moist storms, and have larger radii of maximum winds relative to their outer radii. Cyclones in all simulations have buoyant cores and tangential winds that decrease with height and radius from a near-surface maximum in the eyewall. Storms over fully dry or nearly dry surfaces appear to have larger convective cores than moist storms, suggesting that the size of their inner convective circulations may limit their intensity. In general, the size of the convecting portion of the circulation seems most plausibly explained by the length scale $v_{p} / f$, rather than by the deformation radius, while the overall size depends not on a single length scale but on a combination of length scales for the inner convecting and outer nonconvecting circulations. Existing theory for vortex structure proposed by Emanuel and Rotunno (2011) and C15 appears to apply relatively well over the whole range of surface moisture explored, particularly for explaining the more rapid decay of the outer circulation as storms become drier. Moist storms appear to have more persistence of variability in their outer size than drier storms, enabling the coexistence of storms of similar structures but different sizes as is found in nature. Finally, a fascinating and unexplained result is the presence of a "no-storms-land," where spontaneous genesis does not occur (at least for the domain sizes and simulation durations used here), but where vortices can nonetheless persist if they have already formed. This nostorms-land is a completely new finding, and extends from surface wetness values of $\beta=0.01-0.3$ as well as surface temperatures of $T_{S}=250-270 \mathrm{~K}$. All of these main qualitative results appear to hold for both methods of drying the surface: reducing the surface wetness $\beta$ and reducing the surface temperature $T_{S}$.

Although our simulations have extensively explored the moist-dry transition, many open questions remain. We have no compelling explanation for the existence of the no-storms-land in either suite of simulations, nor is it clear what parameters delineate its boundaries. Its 
occurrence in both sets of simulations suggests that the process of cyclogenesis is discontinuous between the moist and dry limits, and that spontaneous cyclogenesis is somehow impeded for moderately dry surfaces. We speculate that its presence may tie to the enhanced rainfall evaporation and surface air cooling that would accompany a nascent cyclone in an atmosphere with a deep dry boundary layer-traces of the role of rainfall evaporation in reducing low-level temperature are seen for the half-dry storm in Fig. 4e. A similar no-stormsland in the warm-cold transition may seem difficult to explain with this hypothesis, as the boundary layer does not dry or deepen much in the warm-cold transition and precipitation evaporation in the subcloud layer appears minimal in the nonrotating cold RCE simulations (Fig. 3d). Cold simulations, however, have a sedimentation flux of cloud ice at the top of the subcloud layer that matches or exceeds the combined precipitation flux of rain, snow, and graupel shown in Fig. 3d, and nearly all of this cloud ice sublimates as it settles through the subcloud layer. Thus, overall precipitation efficiency is reduced in cold simulations, similar to the semidry simulations, and a similar argument for subcloud-layer evaporation impeding genesis might apply. Other speculative factors that might influence the no-storms-land include the relative depths of the boundary layer and free troposphere, the bulk static stability of the troposphere, and the differing vertical structure of buoyancy anomalies in a weak-amplitude convective vortex. Although most real-world storms are thought to be traceable to preexisting dynamic or thermodynamic disturbances, our generally poor understanding of tropical cyclogenesis suggests that this no-storms-land region of parameter space may be worthy of careful future study.

We hypothesize that the intensity of dry storms is limited by the large size of the inner convective core of dry cyclones (relative to $v_{p} / f$ ), but we have not rigorously tested this hypothesis. We also lack an explanation for why the convective cores of dry cyclones become so large. Mrowiec et al. (2011) found that dry axisymmetric vortices attained peak winds close to their potential intensities, but their vortex core size was constrained to be much smaller than $v_{p} / f$ due to their choice of domain size. The wind fields of our simulated dry storms feature strong azimuthal asymmetries, which could contribute to weakening dry storms either directly, by smearing out a ring of peak winds across a range of radii, or indirectly, by contributing to the growth of the inner core of the cyclone. The mechanisms limiting the intensity of semidry storms could be different than those that weaken fully dry storms, as the inner-core radius, vertical structure of the warm core, and strength of azimuthal asymmetries all differ for semidry storms relative to fully dry ones.
We have conducted simulations with varied Coriolis parameter for fully moist and dry limits (not shown in this paper), which suggest that our main results are similar for smaller values of $f$ than used here. Future work will discuss how dry-TC worlds relate to previous work on rotating dry convection, and particularly will explore the role of the convective Rossby number in potentially altering the behavior of dry-TC worlds at higher $f$ than used in this study. As an additional pathway to connect with work on rotating dry convection, we speculate that dry tropical cyclones might conceivably be produced in a rotating-tank laboratory environment under the right combination of parameters (e.g., Fernando et al. 1991). We plan to explore this possibility in future work.

Together with the work of Mrowiec et al. (2011), this study strongly challenges the conventional wisdom that moisture is essential for tropical cyclone dynamics. Although troposphere-spanning dry convection occurs nowhere in Earth's present climate, purely dry fluid dynamics in rotating RCE over a surface of fixed temperature appear sufficient to spontaneously generate large-scale coherent cyclones. This work points to the benefits of using numerical models as an experimental tool to push beyond the parameter regimes found in Earth's present-day climate, to test existing theories, better understand our own world, and explore possible dynamics of other worlds. We hope that others agree, and that dry and semidry tropical cyclones help to lift the veil of moist complexity and afford a new window into the mysteries of tropical cyclones.

Acknowledgments. Timothy W. Cronin was supported by an Ally of Nature Fund Award. We thank Kerry Emanuel, Morgan O'Neill, and Da Yang for useful conversations, and Marat Khairoutdinov for freely providing SAM. Namelist and sounding files for SAM, as well as processed model output files including statistics of surface winds and azimuthal-average structure files for each tracked cyclone, are available at https://www.dropbox.com/sh/66h0nqgs1ptj8ue/ AABlzhq4v0WUQ7HAU_Gjradna?dl=0. Raw SAM output is available from the authors upon email request (twcronin@mit.edu). Both authors contributed equally to this work.

\section{REFERENCES}

Abarca, S. F., and M. T. Montgomery, 2014: Are eyewall replacement cycles governed largely by axisymmetric balance dynamics? J. Atmos. Sci., 71, 3723-3738, https://doi.org/10.1175/JAS-D14-0018.1.

Allen, M. R., and W. J. Ingram, 2002: Constraints on future changes in climate and the hydrological cycle. Nature, 419, 228-232, https://doi.org/10.1038/nature01092. 
Bister, M., and K. A. Emanuel, 1998: Dissipative heating and hurricane intensity. Meteor. Atmos. Phys., 65, 233-240, https:// doi.org/10.1007/BF01030791.

Bjerknes, J., 1938: Saturated-adiabatic ascent of air through dry-adiabatically descending environment. Quart. J. Roy. Meteor. Soc., 64, 325-330.

Bony, S., B. Stevens, D. Coppin, T. Becker, K. A. Reed, A. Voigt, and B. Medeiros, 2016: Thermodynamic control of anvil cloud amount. Proc. Natl. Acad. Sci. USA, 113, 8927-8932, https:// doi.org/10.1073/pnas.1601472113.

Charney, J. G., and A. Eliassen, 1964: On the growth of the hurricane depression. J. Atmos. Sci., 21, 68-75, https://doi.org/ 10.1175/1520-0469(1964)021<0068:OTGOTH>2.0.CO;2.

Chavas, D. R., and K. Emanuel, 2014: Equilibrium tropical cyclone size in an idealized state of axisymmetric radiative-convective equilibrium. J. Atmos. Sci., 71, 1663-1680, https://doi.org/ 10.1175/JAS-D-13-0155.1.

, and N. Lin, 2016: A model for the complete radial structure of the tropical cyclone wind field. Part II: Wind field variability. J. Atmos. Sci., 73, 3093-3113, https://doi.org/10.1175/JAS-D15-0185.1.

— - _ - and K. Emanuel, 2015: A model for the complete radial structure of the tropical cyclone wind field. Part I: Comparison with observed structure. J. Atmos. Sci., 72, 3647-3662, https:// doi.org/10.1175/JAS-D-15-0014.1.

_,$\ldots$, W. Dong, and Y. Lin, 2016: Observed tropical cyclone size revisited. J. Climate, 29, 2923-2939, https://doi.org/ 10.1175/JCLI-D-15-0731.1.

_ , K. A. Reed, and J. A. Knaff, 2017: Physical understanding of the tropical cyclone wind-pressure relationship. Nat. Commun., 8, 1360, https://doi.org/10.1038/s41467-017-01546-9.

Cronin, T. W., and A. A. Wing, 2017: Clouds, circulation, and climate sensitivity in a radiative-convective equilibrium channel model. J. Adv. Model. Earth Syst., 9, 2883-2905, https:// doi.org/10.1002/2017MS001111.

Emanuel, K. A., 1986: An air-sea interaction theory for tropical cyclones. Part I: Steady-state maintenance. J. Atmos. Sci., 43, 585-605, https://doi.org/10.1175/1520-0469(1986)043<0585: AASITF $>2.0 . \mathrm{CO} ; 2$.

- 2004: Tropical cyclone energetics and structure. Atmospheric Turbulence and Mesoscale Meteorology, Cambridge University Press, 165-191.

_- and R. Rotunno, 2011: Self-stratification of tropical cyclone outflow. Part I: Implications for storm structure. J. Atmos. Sci., 68, 2236-2249, https://doi.org/10.1175/JAS-D-10-05024.1.

Fernando, H. J., R.-R. Chen, and D. L. Boyer, 1991: Effects of rotation on convective turbulence. J. Fluid Mech., 228, 513-547, https://doi.org/10.1017/S002211209100280X.

Held, I. M., 2005: The gap between simulation and understanding in climate modeling. Bull. Amer. Meteor. Soc., 86, 1609-1614, https://doi.org/10.1175/BAMS-86-11-1609.

Kepert, J. D., 2013: How does the boundary layer contribute to eyewall replacement cycles in axisymmetric tropical cyclones? J. Atmos. Sci., 70, 2808-2830, https://doi.org/10.1175/JAS-D13-046.1.

Khairoutdinov, M. F., and D. A. Randall, 2003: Cloud resolving modeling of the ARM summer 1997 IOP: Model formulation, results, uncertainties, and sensitivities. J. Atmos. Sci., 60, 607-625, https:// doi.org/10.1175/1520-0469(2003)060<0607:CRMOTA > 2.0.CO;2. , and K. Emanuel, 2013: Rotating radiative-convective equilibrium simulated by a cloud-resolving model. J. $A d v$. Model. Earth Syst., 5, 816-825, https://doi.org/10.1002/ 2013MS000253.
Malkus, J. S., and H. Riehl, 1960: On the dynamics and energy transformations in steady-state hurricanes. Tellus, 12, 1-20, https://doi.org/10.3402/tellusa.v12i1.9351.

Merrill, R. T., 1984: A comparison of large and small tropical cyclones. Mon. Wea. Rev., 112, 1408-1418, https://doi.org/10.1175/ 1520-0493(1984)112<1408:ACOLAS>2.0.CO;2.

Montgomery, M., M. Nicholls, T. Cram, and A. Saunders, 2006: A vortical hot tower route to tropical cyclogenesis. J. Atmos. Sci., 63, 355-386, https://doi.org/10.1175/JAS3604.1.

Mrowiec, A., S. Garner, and O. Pauluis, 2011: Axisymmetric hurricane in a dry atmosphere: Theoretical framework and numerical experiments. J. Atmos. Sci., 68, 1607-1619, https://doi.org/10.1175/ 2011JAS3639.1.

Muller, C. J., and D. M. Romps, 2018: Acceleration of tropical cyclogenesis by self-aggregation feedbacks. Proc. Natl. Acad. Sci. USA, 115, 2930-2935, https://doi.org/10.1073/pnas.1719967115.

Ooyama, K., 1969: Numerical simulation of the life cycle of tropical cyclones. J. Atmos. Sci., 26, 3-40, https://doi.org/10.1175/15200469(1969)026<0003:NSOTLC >2.0.CO;2.

Oroud, I. M., 1994: Evaluation of saturation vapor pressure over hypersaline water bodies at the southern edge of the Dead Sea, Jordan. Sol. Energy, 53, 497-503, https://doi.org/10.1016/ 0038-092X(94)90129-P.

Pauluis, O., and I. M. Held, 2002: Entropy budget of an atmosphere in radiative-convective equilibrium. Part I: Maximum work and frictional dissipation. J. Atmos. Sci., 59, 125-139, https:// doi.org/10.1175/1520-0469(2002)059<0125:EBOAAI $>2.0 . C O ; 2$.

Rafkin, S. C., 2009: A positive radiative-dynamic feedback mechanism for the maintenance and growth of Martian dust storms. J. Geophys. Res., 114, E01009, https://doi.org/ 10.1029/2008JE003217.

Reed, K. A., and D. R. Chavas, 2015: Uniformly rotating global radiative-convective equilibrium in the Community Atmosphere Model, version 5. J. Adv. Model. Earth Syst., 7, 1938-1955, https:// doi.org/10.1002/2015MS000519.

Rennó, N. O., M. L. Burkett, and M. P. Larkin, 1998: A simple thermodynamical theory for dust devils. J. Atmos. Sci., 55, 3244-3252, https://doi.org/10.1175/1520-0469(1998)055<3244: ASTTFD $>2.0 . \mathrm{CO} ; 2$

Salhotra, A. M., E. E. Adams, and D. R. F. Harleman, 1985: Effect of salinity and ionic composition on evaporation: analysis of Dead Sea evaporation pans. Water Resour. Res., 21, 1336-1344, https://doi.org/10.1029/WR021i009p01336.

Schenkel, B. A., N. Lin, D. R. Chavas, M. Oppenheimer, and A. Brammer, 2017: Evaluating outer tropical cyclone size in reanalysis datasets using QuikSCAT data. J. Climate, 30, 8745-8762, https://doi.org/10.1175/JCLI-D-17-0122.1.

Schubert, W. H., M. T. Montgomery, R. K. Taft, T. A. Guinn, S. R. Fulton, J. P. Kossin, and J. P. Edwards, 1999: Polygonal eyewalls, asymmetric eye contraction, and potential vorticity mixing in hurricanes. J. Atmos. Sci., 56, 1197-1223, https:// doi.org/10.1175/1520-0469(1999)056<1197:PEAECA >2.0.CO;2.

Shea, D. J., and W. M. Gray, 1973: The hurricane's inner core region. I. Symmetric and asymmetric structure. J. Atmos. Sci., 30, 1544-1564, https://doi.org/10.1175/1520-0469(1973) $030<1544$ :THICRI $>2.0$. CO 2 .

Stern, D. P., J. L. Vigh, D. S. Nolan, and F. Zhang, 2015: Revisiting the relationship between eyewall contraction and intensification. J. Atmos. Sci., 72, 1283-1306, https://doi.org/10.1175/ JAS-D-14-0261.1.

Tang, B., and K. Emanuel, 2010: Midlevel ventilation's constraint on tropical cyclone intensity. J. Atmos. Sci., 67, 1817-1830, https://doi.org/10.1175/2010JAS3318.1. 
Tokano, T., 2013: Are tropical cyclones possible over Titan's polar seas? Icarus, 223, 766-774, https://doi.org/10.1016/ j.icarus.2013.01.023.

Williams, G. J., R. K. Taft, B. D. McNoldy, and W. H. Schubert, 2013: Shock-like structures in the tropical cyclone boundary layer. J. Adv. Model. Earth Syst., 5, 338-353, https://doi.org/ 10.1002/jame.20028.

Wing, A. A., S. J. Camargo, and A. H. Sobel, 2016: Role of radiativeconvective feedbacks in spontaneous tropical cyclogenesis in idealized numerical simulations. J. Atmos. Sci., 73, 2633-2642, https://doi.org/10.1175/JAS-D-15-0380.1.

Yanai, M., 1964: Formation of tropical cyclones. Rev. Geophys., 2, 367-414, https://doi.org/10.1029/RG002i002p00367.

Zhou, W., I. M. Held, and S. T. Garner, 2014: Parameter study of tropical cyclones in rotating radiative-convective equilibrium with column physics and resolution of a $25-\mathrm{km}$ GCM. J. Atmos. Sci., 71, 1058-1069, https://doi.org/10.1175/ JAS-D-13-0190.1. 